\title{
Performance analysis of interference-limited dual-hop multiple antenna AF relaying systems with feedback delay
}

\author{
Jinlong Wang ${ }^{1}$, Yuzhen Huang ${ }^{1 *}$, Caijun Zhong ${ }^{2,3}$, Fawaz Al-Qahtani ${ }^{4}$, Qihui Wu ${ }^{1}$ and Yunpeng Cheng ${ }^{1}$
}

\begin{abstract}
In this paper, we present a comprehensive performance analysis of dual-hop multiple antenna channel state information (CSI) assisted amplify-and-forward (AF) relaying systems over Rayleigh fading channels employing arbitrary transmit antenna selection (TAS) and receiver maximum ratio combining (MRC) with feedback delay in the presence of co-channel interference (CCl) at both the relay and destination. Specifically, an upper bound on the cumulative distribution function (CDF) of the end-to-end signal-to-interference ratio (SIR) is proposed, based on which closed-form expressions for the outage probability and the average symbol error rate (SER) are derived. To gain further insights, simple and high informative expressions for the outage probability and the average SER are obtained at the high SIR regime, which readily enable us to characterize the achievable diversity order and coding gain of the system. Moreover, we present new analytical upper and lower bounds for the ergodic capacity of the system, which apply to the system with arbitrary number of antennas, $\mathrm{CCl}$ and feedback delay at any SIRs. Finally, to minimize the outage probability of the system, an optimum power allocation scheme is devised under the total transmission power constraint between the source and the relay. The findings suggest that the feedback delay limits the diversity order to one, while the $\mathrm{CCl}$ degrades the outage performance by affecting the coding gain of the system.
\end{abstract}

Keywords: Amplify-and-forward (AF) relaying; Multiple antenna system; Co-channel interference (CCI); Outage probability; Symbol error rate (SER); Ergodic capacity; Power allocation

\section{Introduction}

Relaying transmission has been proposed as a promising technique to improve the coverage and throughput of wireless communication systems [1]. Among the various relaying protocols, the most two common relaying schemes are amplify-and-forward (AF) and decode-andforward (DF). In particular, the AF relaying scheme has received significant attention due to its low implementation complexity. Depending on the availability of channel state information (CSI) at the relay, the AF relaying scheme generally falls into two categories, i.e., variable gain relaying [2] and fixed gain relaying [3]. Over the last decades, a significant amount of effort has been devoted to investigate the key performance measures such as

\footnotetext{
${ }^{*}$ Correspondence: yzh_huang@sina.com

${ }^{1}$ College of Communications Engineering, PLA University of Science and

Technology, Nanjing, 210007, China

Full list of author information is available at the end of the article
}

outage probability, average symbol error rate (SER), and ergodic capacity for AF relaying schemes over various fading channels [4-8].

Nevertheless, all the above works assume that the relaying systems operate in a noise-limited environment. However, future wireless communication networks tend to operate in an interference-limited environment due to aggressive frequency reuse. Motivated by this observation, many works have investigated the effect of co-channel interference $(\mathrm{CCI})$ on the performance of the relaying systems in a variety of different scenarios. In [9], the authors proposed a relay selection algorithm for dualhop CSI-assisted AF relaying networks in the presence of interference at the relay. In [10], the outage probabilities for both AF and DF relaying systems with noisy relay and interference-limited destination were derived. In [11], the authors investigated the outage performance of dual-hop fixed-gain relaying systems in the presence of interference at both the relay and destination and pointed

\section{Springer}

(c) 2013 Wang et al: licensee Springer. This is an Open Access article distributed under the terms of the Creative Commons Attribution License (http://creativecommons.org/licenses/by/2.0), which permits unrestricted use, distribution, and reproduction in any medium, provided the original work is properly cited. 
out that the worst outage performance occurred when the power of interference at the relay and destination was equal. In $[12,13]$, the authors investigated the joint effects of imperfect channel estimation and CCI on the performance of dual-hop relaying systems, respectively. Most recently, some works addressed the case with Nakagami$m$ fading [14-18]. In [14], the authors studied the outage probability and the average BER of dual-hop fixed-gain AF relaying systems with a single interferer in Nakagami- $m$ fading channels. In [15], the authors considered the performance of interference-limited dual-hop CSI-assisted AF systems in Nakagami- $m$ fading channels. In [16], the outage probability of multi-branch dual-hop DF cooperative relaying systems with CCI over Nakagami- $m$ fading channels was analyzed. In [17], the authors derived the exact closed-form expression for the outage probability of multi-hop transmission systems with AF and DF relaying protocols in Nakagami- $m$ fading channels in the presence of CCI and made a comprehensive comparison between the two relaying protocols. In [18], the authors analyzed the ergodic capacity of dual-hop CSI-assisted AF relaying system with both CCI at the relay and destination.

While these prior works have improved our understanding on the impact of CCI on the performance of dualhop relaying systems, the key limitation of these works is that they all consider a single antenna relaying system. With the fact that the multiple antenna technique will be adopted as one of the key enabling technologies for the next generation wireless communication systems, the importance of understanding the fundamental performance of multiple antenna relaying systems becomes increasingly evident [19-22]. In [19], the outage probability and the average SER were analyzed in a two-hop multiple-input multiple-output (MIMO) relaying network using transmit antenna selection with receiver maximalratio combining (TAS/MRC). In [20], a unified framework for the average SER of distributed TAS/MRC in MIMO relaying networks was proposed. In [21], two attractive MIMO protocols, i.e., TAS/MRC and transmit antenna selection with receive selection combining (TAS/SC), were analyzed in a dual-hop AF relaying network with respect to the average SER. In [22], a low-complexity protocol that guaranteed a two-fold diversity, i.e., multiantenna diversity via TAS/MRC and multiuser diversity via opportunistic scheduling, was proposed in multiuser AF relaying networks. However, it is worth pointing out that these aforementioned works also assume the noiselimited scenario; hence, the impact of CCI on the performance of dual-hop multiple antenna AF relaying systems has not been well understood. Recently, only a few analytical results concerning the performance of dual-hop multiple antenna AF relaying systems in the presence of $\mathrm{CCI}$ are available in the literature [23-25]. In [23], the performance of dual-hop fixed-gain AF relaying systems with a single Rayleigh interferer at the relay was investigated under the special case, where only one of the nodes is equipped with multiple antennas. In [24], the outage performance of a dual-hop fixed-gain AF MIMO relaying network using maximum ratio transmission and maximum ratio combining (MRT/MRC) in the presence of interference was analyzed. In parallel, [25] investigated the effect of feedback delay on the outage probability and the average SER of multiple antenna AF relaying systems employing MRT/MRC.

In this paper, different from these prior works, we consider a dual-hop multiple antenna CSI-assisted AF relaying system applying arbitrary TAS/MRC in the presence of interference at both the relay and destination. Furthermore, the CSI feedback delay of the first-hop link is also taken into consideration. For such practical system model, we pursue a comprehensive analysis of the joint effects of feedback delay and multiple antennas on the performance of the system. The main contributions of the paper are summarized as follows:

- We propose an upper bound of the equivalent endto-end signal-to-interference ratio (SIR) of the system and derive its cumulative distribution function (CDF).

- With the help of the CDF of the proposed upper bound, we investigate the joint effects of number of antennas, $\mathrm{CCI}$, and feedback delay on the system performance in terms of the outage probability and the average SER. Specifically, we present closed-form expressions for the outage probability and the average SER, which provide a fast and efficient means to evaluate the performance of the system. In addition, we look into the asymptotic high SIR regime and characterize the key performance measures such as diversity order and coding gain. Our findings suggest that the impact of feedback delay on the performance of the system is significant as it limits the diversity order to one regardless of the number of antennas at the source and destination. On the other hand, the impact of CCI on the performance of the system is less significant, since it does not reduce the diversity order, but affects the coding gain of the system.

- We also present new analytical upper and lower ergodic capacity bounds for interference-limited dual-hop multiple antenna CSI-assisted AF relaying systems with feedback delay. The proposed bounds apply to arbitrary number of antennas, CCI, and feedback delay at any SIR. Moreover, they involve only standard mathematical functions and therefore can be easily and efficiently evaluated.

- We investigate the optimum power allocation scheme to minimize the asymptotic outage probability of the system under the constraint that the total transmit power of the source and relay is fixed, and present 
closed-form power allocation expressions. Simulation results demonstrate that the optimum power allocation scheme achieves better coding gain compared to the equal power allocation scheme.

The remainder of the paper is organized as follows. Section 2 introduces the system model. In Section 3, we derive the upper bound of the equivalent end-to-end SIR and investigate the outage probability, average SER, and ergodic capacity of the system. In Section 4, the optimum power allocation scheme is proposed to minimize the outage performance of the system. Numerical results are presented in Section 5. Finally, Section 6 concludes the paper.

\section{System model}

We consider a dual-hop CSI-assisted AF relaying system in the presence of multiple interference at both the relay and destination as illustrated in Figure 1, where the source $S$ communicates with the destination $D$ with the help of an intermediate relay node $R$. The source $S$ is equipped with $N_{\mathrm{t}}$ antennas and the destination $D$ is equipped with $N_{\mathrm{r}}$ antennas, whereas the relay $R$ only has a single antenna. The $S \rightarrow R$ channel vector is denoted by $\mathbf{h}_{s r}=\left[h_{1 r}, \ldots, h_{N_{\mathrm{t}} r}\right]^{T}$, and its entries follow independent and identically distributed (i.i.d.) Rayleigh fading with parameters $\mathrm{E}\left[\left|h_{i r}\right|^{2}\right]=\lambda_{s r}$, while the $R \rightarrow D$ channel vector is denoted by $\mathbf{h}_{r d}=\left[h_{1 d}, \ldots, h_{N_{\mathrm{r}} d}\right]^{T}$, and its entries follow i.i.d. Rayleigh fading with parameters $\mathrm{E}\left[\left|h_{j r}\right|^{2}\right]=\lambda_{r d}$. To reduce the cost and the complexity of system design while maintaining satisfying performance, we adopt TAS algorithm in this paper, in which a single antenna is selected based on the feedback of CSI from the relay $R$. We consider the general scenario where the $k$ th worst antenna is selected ${ }^{\mathrm{a}}$. Clearly, $k=N$ corresponding to the case where the best antenna is selected. In addition, due to imperfect feedback link, the feedback delay of CSI about the first link is also considered.

The entire communication between the source $S$ and the destination $D$ consists of two orthogonal phases. In the first phase, the source $S$ transmits the desired signal to the relay $R$ with an average transmit power $P_{S}$, which is impaired by $N_{I_{1}}$ interfering signals with an average power of $P_{i r}, i \in\left\{1, \cdots, N_{I_{1}}\right\}$. Assuming the interferencelimited environment, the received signal at the relay can be expressed as

$$
y_{R}=\sqrt{P_{s}} h_{(k) r} x_{s}+\sum_{i=1}^{N_{I_{1}}} \sqrt{P_{i r}} g_{i r} x_{i r}
$$

where $h_{(k) r}$ is the channel coefficient between the selected $k$ th worst antenna and the relay node, $x_{s}$ is the source symbol with $\mathrm{E}\left[\left|x_{s}\right|^{2}\right]=1, x_{i r}$ is the $i$ th interference symbol satisfying $\mathrm{E}\left[\left|x_{i r}\right|^{2}\right]=1$, and $g_{i r}$ is the corresponding interference channel link, whose amplitude follows Rayleigh distribution with parameter $\lambda_{i r}$.

In the second phase, the relay $R$ forwards the received signal to the destination $D$ after applying a gain factor $G$. As in the previous literature [3,26,27], the relay gain $G$ is given by

$$
G=\sqrt{P_{r} /\left(P_{s}\left|h_{(k) r}\right|^{2}+\sum_{i=1}^{N_{I_{1}}} P_{i r}\left|g_{i r}\right|^{2}\right)}
$$

where $P_{r}$ denotes the transmit power at the relay $R$. Therefore, the received signal at the destination $D$, after MRC processing, is given by

$$
\begin{aligned}
y_{D}= & G \mathbf{w}^{T} \mathbf{h}_{r d}\left(\sqrt{P_{s}} h_{(k) r} x_{s}+\sum_{i=1}^{N_{I_{1}}} \sqrt{P_{i r}} g_{i r} x_{i r}\right) \\
& +\mathbf{w}^{T} \sum_{j=1}^{N_{I_{2}}} \sqrt{P_{j d}} \mathbf{g}_{j d} x_{j d},
\end{aligned}
$$

where $\mathbf{w}=\frac{\mathbf{h}_{r d}}{\left\|\mathbf{h}_{r d}\right\|_{F}}$ is the $N_{\mathrm{r}} \times 1$ weight vector with $\|\cdot\|_{F}$ being the Frobenius norm, $N_{I_{2}}$ is the number of interferers at the destination, $P_{j d}$ is the transmit power of the $j$ th interference at the destination, $x_{j d}$ is the $j$ th interference symbol at the destination with a unit energy, $\mathbf{g}_{j d}=\left[g_{j 1 d}, \ldots, g_{j N_{\mathrm{r}} d}\right]^{T}$ is the $j$ th interference channel vector for $j \in\left\{1, \ldots, N_{I_{2}}\right\}$, and the amplitude of its entries follows i.i.d. Rayleigh fading with parameter $\mathrm{E}\left[\left|g_{j m d}\right|^{2}\right]=\lambda_{j d}$ for $m \in\left\{1, \ldots, N_{\mathrm{r}}\right\}$.

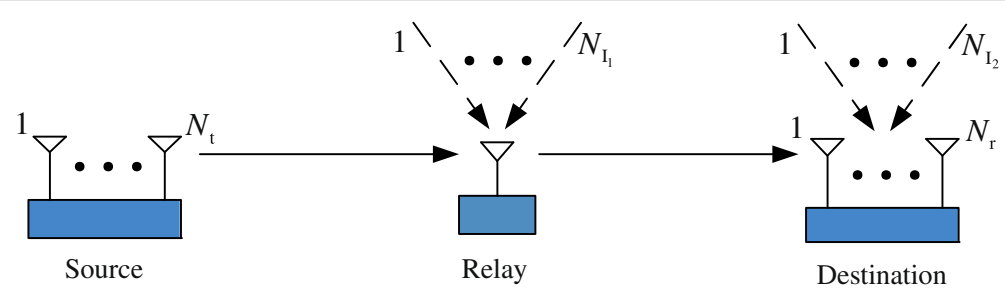

Figure 1 System model. 
Defining $f_{j d}=\mathbf{w}^{T} \mathbf{g}_{j d}$, and using the results reported in $[23,24]$, it can be easily shown that $\left|f_{j d}\right|$ follows the Rayleigh distribution with parameters $\mathrm{E}\left[\left|f_{j d}\right|^{2}\right]=\lambda_{j d}$ for $j \in\left\{1, \ldots, N_{I_{2}}\right\}$. To this end, after some mathematical manipulations, the end-to-end SIR can be expressed as

$$
\gamma=\frac{\tilde{\gamma}_{1(k)} \gamma_{2}}{\tilde{\gamma}_{1(k)} \gamma_{I_{2}}+\gamma_{2} \gamma_{I_{1}}+\gamma_{I_{1}} \gamma_{I_{2}}}
$$

where $\tilde{\gamma}_{1(k)}=P_{s}\left|h_{(k) r}\right|^{2}, \gamma_{2}=P_{r}\left\|\mathbf{h}_{r d}\right\|_{F}^{2}, \gamma_{I_{1}}=\sum_{i=1}^{N_{I_{1}}} P_{i r}\left|g_{i r}\right|^{2}$, and $\gamma_{I_{2}}=\sum_{j=1}^{N_{I_{2}}} P_{j d}\left|f_{j d}\right|^{2}$.

Let $\gamma_{1(1)} \leq \cdots \leq \gamma_{1\left(N_{\mathrm{t}}\right)}$ be the order statistics obtained by arranging $\gamma_{1(k)}$ for $k \in\left\{1, \cdots, N_{\mathrm{t}}\right\}$ in an increasing order, where $\gamma_{1(k)}$ denotes the instantaneous transmit power between the $k$ th worst antenna and the relay without feedback delay, then $\widetilde{\gamma}_{1(k)}$ is the $\tau$ time delayed version of $\gamma_{1(k)}$. According to [28-30], the relation between $\tilde{\gamma}_{1(k)}$ and $\gamma_{1(k)}$ can be modeled as

$$
\tilde{\gamma}_{1(k)}=\sqrt{\rho} \gamma_{1(k)}+\sqrt{1-\rho} \omega_{1(k)}
$$

where $\left|\omega_{1(k)}\right|^{2}$ is the chi-square distributed random variable (RV) having the same variance as $\gamma_{1(k)}$, and $\rho$ is the correlation coefficient between $\widetilde{\gamma}_{1(k)}$ and $\gamma_{1(k)}$ which, according to Jakes' autocorrelation model [30], can be expressed as

$$
\rho=\left[J_{0}\left(2 \pi f_{d} \tau\right)\right]^{2}
$$

where $J_{0}(\cdot)$ denotes the zeroth-order Bessel function of the first kind ([31], eq. (8.411)), and $f_{d}$ is the maximum Doppler frequency. The probability density function (PDF) of $\widetilde{\gamma}_{1(k)}$ is given by

$$
f_{\widetilde{\gamma}_{1(k)}}(x)=\int_{0}^{\infty} f_{\widetilde{\gamma}_{1(k)} \mid \gamma_{1(k)}}(x \mid y) f_{\gamma_{1(k)}}(y) d y
$$

where $f_{\widetilde{\gamma}_{1(k)} \mid \gamma_{1(k)}}(x \mid y)=f_{\widetilde{\gamma}_{1(l)}, \gamma_{1(l)}}(x, y) / f_{\gamma_{1(l)}}(y)$ is the PDF of $\widetilde{\gamma}_{1(k)}$ conditioned on $\gamma_{1(k)}$. Since $\tilde{\gamma}_{1(l)}$ and $\gamma_{1(k)}$ are the two correlated exponentially distributed RVs, the joint PDF of $\tilde{\gamma}_{1(l)}$ and $\gamma_{1(k)}$ can be expressed as [32]

$$
f_{\widetilde{\gamma}_{1(l)}, \gamma_{1(l)}}(x, y)=\frac{1}{(1-\rho) \bar{\gamma}_{1}^{2}} e^{-\frac{(x+y)}{(1-\rho) \bar{\gamma}_{1}}} I_{0}\left(\frac{2 \sqrt{\rho x y}}{(1-\rho) \bar{\gamma}_{1}}\right),
$$

where $\bar{\gamma}_{1}=P_{s} \lambda_{s r}$ denotes the average power of the first hop, and $I_{0}(\cdot)$ denotes the modified Bessel function of the first kind ([31], eq.(8.406.1)). On the other hand, the PDF of $\gamma_{1(k)}$ can be derived as

$$
f_{\gamma_{1(k)}}(y)=k\left(\begin{array}{c}
N_{\mathrm{t}} \\
k
\end{array}\right)\left[F_{\gamma_{1(l)}}(y)\right]^{k-1}\left[1-F_{\gamma_{1(l)}}(y)\right]^{N_{\mathrm{t}}-k} f_{\gamma_{1(l)}}(y),
$$

where $f_{\gamma_{1(l)}}(y)=1 / \bar{\gamma}_{1} e^{-y / \bar{\gamma}_{1}}$ and $F_{\gamma_{1(l)}}(y)=1-e^{-y / \bar{\gamma}_{1}}$. Hence, substituting (8) and (9) into (7) and utilizing the approach in [33], we have

$$
f_{\tilde{\gamma}_{1}(k)}(x)=k\left(\begin{array}{c}
N_{\mathrm{t}} \\
k
\end{array}\right) \sum_{n=0}^{k-1} \frac{(-1)^{n}}{\bar{\gamma}_{1}}\left(\begin{array}{c}
k-1 \\
n
\end{array}\right) \frac{\alpha}{\beta} e^{-\frac{\alpha}{\bar{\gamma}_{1}} x},
$$

where $\alpha=\frac{\beta}{\left(N_{\mathrm{t}}-k+n\right)(1-\rho)+1}$ and $\beta=N_{\mathrm{t}}-k+n+1$. To this end, the corresponding CDF of $\tilde{\gamma}_{1(k)}$ can be deduced from (10) as

$$
F_{\widetilde{\gamma}_{1(k)}}(x)=1-k\left(\begin{array}{c}
N_{\mathrm{t}} \\
k
\end{array}\right) \sum_{n=0}^{k-1} \frac{(-1)^{n}}{\beta}\left(\begin{array}{c}
k-1 \\
n
\end{array}\right) e^{-\frac{\alpha}{\bar{\gamma}_{1}} x} .
$$

\section{End-to-end performance analysis}

In this section, we perform a comprehensive performance analysis of the system under consideration by presenting closed-form analytical expressions for key performance measures, i.e., the outage probability, the average SER, and the ergodic capacity. We start by characterizing the statistics of the upper bound of the end-to-end SIR. Based on which, the closed-form approximations for the outage probability and the average SER are obtained. Moreover, to gain more insights, we look into the asymptotic high SIR regime and present simple analytical expressions for the outage probability and the average SER. Finally, we investigate the ergodic capacity of the system and propose tight upper and lower bounds on the ergodic capacity of the system.

\subsection{Upper bound of the end-to-end SIR}

In general, an exact analysis of the statistics of the endto-end SIR shown in (4) is very challenging. Hence, to circumvent this difficulty and obtain useful analytical results to evaluate the performance of the system, we first propose the following upper bound for the end-to-end SIR:

$$
\gamma \leq \gamma_{\text {up }}=\min \left(\frac{\tilde{\gamma}_{1(k)}}{\gamma_{I_{1}}}, \frac{\gamma_{2}}{\gamma_{I_{2}}}\right) .
$$

It is worth pointing out that the same bounding technique has been widely adopted in the performance analysis of various relaying systems (see [12] and references therein). 
In addition, it has been demonstrated that this upper bound is in general very tight, especially in the high SIR regime.

To this end, to analyze the outage and SER performance of the system, the statistical behavior of the SIR upper bound $\gamma_{\text {up }}$ is required, which is given in the following theorem:

Theorem 1. The exact CDF of $\gamma_{\mathrm{up}}$ is given by

$$
\begin{aligned}
F_{\gamma_{\text {up }}}(x)=1 & -k\left(\begin{array}{c}
N_{\mathrm{t}} \\
k
\end{array}\right) \sum_{n=0}^{k-1} \frac{(-1)^{n}}{\beta}\left(\begin{array}{c}
k-1 \\
n
\end{array}\right) \sum_{i=1}^{N_{I_{1}}} \frac{\eta_{i} \bar{\gamma}_{1}}{\alpha x \bar{\gamma}_{3 i}+\bar{\gamma}_{1}} \\
& \times\left[1-\sum_{j=1}^{N_{I_{2}}} \zeta_{j}\left(\frac{x \bar{\gamma}_{4 j}}{x \bar{\gamma}_{4 j}+\bar{\gamma}_{2}}\right)^{N_{\mathrm{r}}}\right] .
\end{aligned}
$$

Proof. From (12), the CDF of $\gamma_{\text {up }}$ can be expressed as

$$
F_{\gamma_{\text {up }}}(x)=1-\left[1-F_{\widetilde{\gamma}_{1(k)} / \gamma_{I_{1}}}(x)\right]\left[1-F_{\gamma_{2} / \gamma_{I_{2}}}(x)\right]
$$

where $F_{\widetilde{\gamma}_{1(k)} / \gamma_{I_{1}}}(x)$ and $F_{\gamma_{2} / \gamma_{I_{2}}}(x)$ are the CDFs of $\tilde{\gamma}_{1(k)} / \gamma_{I_{1}}$ and $\gamma_{2} / \gamma_{I_{2}}$, respectively. As all the links undergo Rayleigh fading, the CDF of $\gamma_{2}$ is given by $F_{\gamma_{2}}(x)=\gamma\left(N_{\mathrm{r}}, x / \bar{\gamma}_{2}\right) /\left(N_{\mathrm{r}}-1\right)$ !, where $\bar{\gamma}_{2}=P_{r} \lambda_{r d}$ is the average power of the second hop and $\gamma(\cdot, \cdot)$ is the lower incomplete Gamma function ([31], eq.(8.350.1)). The PDFs of $\gamma_{I_{1}}$ and $\gamma_{I_{2}}$ are given by [25]

$$
\begin{aligned}
& f_{\gamma_{I_{1}}}(x)=\sum_{i=1}^{N_{I_{1}}} \frac{\eta_{i}}{\bar{\gamma}_{3 i}} e^{-x / \bar{\gamma}_{3 i},} \\
& f_{\gamma_{I_{2}}}(x)=\sum_{j=1}^{N_{I_{2}}} \frac{\zeta_{j}}{\bar{\gamma}_{4 j}} e^{-x / \bar{\gamma}_{4 j},}
\end{aligned}
$$

where $\bar{\gamma}_{3 i}=P_{i r} \lambda_{i r}$ and $\bar{\gamma}_{4 j}=P_{j d} \lambda_{j d}$ are the average power of the $i$ th interference at the relay and the $j$ th interference at the destination, respectively. In this paper, we assume that $\bar{\gamma}_{3 i}=P_{i r} \lambda_{i r}, i=1, \cdots, N_{I_{1}}$, and $\bar{\gamma}_{4 j}=P_{j d} \lambda_{j d}, j=$ $1, \cdots, N_{I_{2}}$ are distinct. $\eta_{i}$ and $\zeta_{j}$ are given by

$$
\begin{gathered}
\eta_{i}=\left[\prod_{u=1, u \neq i}^{N_{I_{1}}} \frac{\bar{\gamma}_{3 u}^{-1}}{\left(s+\bar{\gamma}_{3 u}^{-1}\right)}\right]_{s=-\bar{\gamma}_{3 i}^{-1}}, \\
\zeta_{j}=\left[\prod_{\theta=1, \theta \neq j}^{N_{I_{2}}} \frac{\bar{\gamma}_{4 \theta}^{-1}}{\left(s+\bar{\gamma}_{4 \theta}^{-1}\right)}\right]_{s=-\bar{\gamma}_{4 j}^{-1}} .
\end{gathered}
$$

Now, by utilizing (11) and (15), $F_{\widetilde{\gamma}_{1(k)} / \gamma_{I_{1}}}(x)$ can be derived, after performing some required integrations, as

$$
\begin{aligned}
F_{\widetilde{\gamma}_{1}(k) / \gamma_{I_{1}}}(x) & =\int_{0}^{\infty} F_{\widetilde{\gamma}_{1(k)}}(x y) f_{\gamma_{I_{1}}}(y) d y \\
& =1-k\left(\begin{array}{c}
N_{\mathrm{t}} \\
k
\end{array}\right) \sum_{n=0}^{k-1} \frac{(-1)^{n}}{\beta}\left(\begin{array}{c}
k-1 \\
n
\end{array}\right) \sum_{i=1}^{N_{I_{1}}} \frac{\eta_{i} \bar{\gamma}_{1}}{\alpha x \bar{\gamma}_{3 i}+\bar{\gamma}_{1}} .
\end{aligned}
$$

Similarly, by employing the CDF of $\gamma_{2}$ and the PDF of $\gamma_{I_{2}}$, and making a change of $\omega=x y$, the CDF of $\gamma_{2} / \gamma_{I_{2}}$ can be computed as

$$
\begin{aligned}
F_{\gamma_{2} / \gamma_{I_{2}}}(x) & =\int_{0}^{\infty} F_{\gamma_{2}}(x y) f_{\gamma_{I_{2}}}(y) d y=\int_{0}^{\infty} \frac{F_{\gamma_{2}}(\omega)}{x} f_{\gamma_{I_{2}}}\left(\frac{\omega}{x}\right) d \omega \\
& =\sum_{j=1}^{N_{I_{2}}} \zeta_{j}\left(\frac{x \bar{\gamma}_{4 j}}{x \bar{\gamma}_{4 j}+\bar{\gamma}_{2}}\right)^{N_{\mathrm{r}}},
\end{aligned}
$$

where we have used [31] eq.(6.451.1) to solve the corresponding integral. Finally, substituting (19) and (20) into (14) yields the desired result.

\subsection{Outage probability}

As an important performance measure of wireless communication systems, the outage probability is defined as the probability that the instantaneous achievable rate falls below a given threshold $R$. Mathematically, the outage probability can be expressed as [32]

$$
P_{\text {out }}(R)=\operatorname{Pr}\left[\frac{1}{2} \log _{2}(1+\gamma)<R\right] \approx F_{\gamma_{\text {up }}}(\Delta),
$$

where $\Delta=2^{2 R}-1$. Hence, with Theorem 1 at hand, the lower bound of outage probability can be directly obtained from (13). Note that the expression (13) only involves the standard function which allows for fast evaluation in popular mathematical software such as Matlab or Mathematica, thereby providing an efficient means to assess the impact of various key system parameters such as number of antennas, $\mathrm{CCI}$, and correlation coefficient on the outage performance of the system.

To gain further understanding on the impact of number of antennas, $\mathrm{CCI}$ and correlation coefficient on the outage performance, we now look into the asymptotic high SIR regime. We find it convenient to treat the following two cases separately. Case 1: interference only (no feedback delay), i.e., $\rho=1$, and Case 2: interference and feedback delay, i.e., $\rho<1$.

For notational convenience, we define $z=\Delta / \bar{\gamma}_{1}$ and $\bar{\gamma}_{2}=\mu \bar{\gamma}_{1}$, where $\mu$ is a positive constant. We first consider the case where only CCI exists in the system, and we have the following important result: 
Corollary 1. With only CCI, i.e., $\rho=1$, the asymptotic outage probability of interference-limited dual-hop multiple antenna CSI-assisted AF relaying systems is given by

$$
P_{\text {out }}^{\infty}(z) \approx \begin{cases}\sum_{i=1}^{N_{I_{1}}} \eta_{i} k\left(\begin{array}{c}
N_{\mathrm{t}} \\
k
\end{array}\right) \sum_{n=0}^{k-1}(-1)^{n+k+1}\left(\begin{array}{c}
k-1 \\
n
\end{array}\right) \beta^{k-1}\left(\bar{\gamma}_{3 i} z\right)^{k}, & k<N_{\mathrm{r}} \\
\sum_{i=1}^{N_{I_{1}}} \eta_{i} \sum_{j=1}^{N_{I_{2}}} \zeta_{j}\left[\left(\frac{\bar{\gamma}_{4 j}}{\mu}\right)^{L}-L\left(\begin{array}{c}
N_{\mathrm{t}} \\
L
\end{array}\right) \sum_{n=0}^{L-1}(-1)^{n+L}\left(\begin{array}{c}
L-1 \\
n
\end{array}\right) \beta^{L-1} \bar{\gamma}_{3 i}^{L}\right] z^{L}, k=N_{\mathrm{r}}=L . \\
\sum_{j=1}^{N_{I_{2}}} \zeta_{j}\left(\frac{\bar{\gamma}_{4 j}}{\mu} z\right)^{N_{\mathrm{r}}}, & k>N_{\mathrm{r}}\end{cases}
$$

\section{Proof. See Appendix 1.}

Corollary 1 suggests the intuitive result that the outage performance of the system depends on the selection order of the transmit antenna, i.e., $k$. Moreover, the impact of CCI on the outage performance varies as the selection order $k$ changes. As we can readily observe, when $k<N_{\mathrm{r}}$, the outage probability is solely affected by the $\mathrm{CCI}$ at the relay and is independent of the $\mathrm{CCI}$ at the destination. Similarly, when $k>N_{\mathrm{r}}$, the outage probability is only affected by the CCI at the destination regardless of the $\mathrm{CCI}$ at the relay. Only for the case $k=N_{\mathrm{r}}$, the impact of CCI at both the relay and destination on the outage probability becomes evident. This phenomena is somehow expected, since in a dual-hop relaying system, the overall outage performance is limited by the worst link.

Now, we look at the case when the system is subjected to both the CCI and feedback delay, and we have the following key result:

Corollary 2. With both the CCI and feedback delay, i.e., $\rho<1$, the asymptotic outage probability of interferencelimited dual-hop multiple antenna CSI-assisted AF relaying systems is given by

$P_{\text {out }}^{\infty}(z) \approx \begin{cases}\sum_{i=1}^{N_{I_{1}}} \eta_{i} \sum_{j=1}^{N_{I_{2}}} \zeta j\left[\frac{\bar{\gamma}_{4 j}}{\mu}+k\left(\begin{array}{c}N_{\mathrm{t}} \\ k\end{array}\right) \sum_{n=0}^{k-1}(-1)^{n+k}\left(\begin{array}{c}k-1 \\ n\end{array}\right) \frac{\alpha \bar{\gamma}_{3 i}}{\beta}\right] & z, N_{\mathrm{r}}=1 \\ \sum_{i=1}^{N_{I_{1}}} \eta_{i} k\left(\begin{array}{c}N_{\mathrm{t}} \\ k\end{array}\right) \sum_{n=0}^{k-1}(-1)^{n}\left(\begin{array}{c}k-1 \\ n\end{array}\right) \frac{\alpha \bar{\gamma}_{3 i}}{\beta} z, & N_{\mathrm{r}}>1\end{cases}$

Proof. Following the same lines as in the proof of Corollary 1 , the asymptotic outage probability when $\rho<1$ can be computed as

$$
\begin{aligned}
P_{\text {out }}^{\infty}(z) \approx & 1-\sum_{i=1}^{N_{I_{1}}} \eta_{i}\left[1+k\left(\begin{array}{c}
N_{\mathrm{t}} \\
k
\end{array}\right) \sum_{n=0}^{k-1}(-1)^{n+1}\left(\begin{array}{c}
k-1 \\
n
\end{array}\right) \frac{\alpha}{\beta} \bar{\gamma}_{3 i} z\right] \\
& \times \sum_{j=1}^{N_{I_{2}}} \zeta_{j}\left[1-\left(\frac{\bar{\gamma}_{4 j}}{\mu} z\right)^{N_{\mathrm{r}}}\right] .
\end{aligned}
$$

To this end, the desired results can be obtained after some algebraic manipulations.

Corollary 2 shows that the achievable diversity order is one when the transmitter only has access to a delayed version of the CSI. Moreover, whether the interference at the destination affects the outage probability of the system depends on the number of antennas employed at the destination. It is worth noting that when the destination has a single antenna, the interference at the destination will affect the outage probability of the system. Otherwise, the interference at the destination has no impact on the outage probability.

\subsection{Average symbol error rate}

In this section, we analyze the average SER of the system, which is another important metric to quantify the performance of wireless communication systems. For a wide range of modulation schemes, the average SER is given by

$$
\bar{P}_{e}=\mathrm{E}[a Q(\sqrt{2 b \gamma})]
$$

where $Q(x)=\frac{1}{\sqrt{2 \pi}} \int_{x}^{\infty} e^{-y^{2} / 2} d y$ is the complementary error function, and $a$ and $b$ are the modulation specific constants. For instance, $a=1, b=1$ for BPSK, $a=1, b=$ 0.5 for BFSK with orthogonal signaling, $a=2(M-1) / M$, $b=3 /\left(M^{2}-1\right)$ for M-PAM, and $a=2, b=\sin ^{2}(\pi / M)$ for M-PSK.

Utilizing the result presented in [34], the average SER can be alternatively computed by

$$
\bar{P}_{e} \approx \frac{a}{2} \sqrt{\frac{b}{\pi}} \int_{0}^{\infty} \frac{\mathrm{e}^{-b x}}{\sqrt{x}} F_{\gamma_{\mathrm{up}}}(x) d x
$$

To this end, with the help of the CDF of the SIR upper bound, we have the following key result: 
Theorem 2. The average SER of interference-limited dual-hop CSI-assisted multiple antenna AF relaying networks with feedback delay is lower bounded by

$$
\bar{P}_{e} \approx \frac{a}{2}-\frac{a k}{2}\left(\begin{array}{c}
N_{\mathrm{t}} \\
k
\end{array}\right) \sum_{n=0}^{k-1}\left(\begin{array}{c}
k-1 \\
n
\end{array}\right) \sum_{i=1}^{N_{I_{1}}} \frac{(-1)^{n} \eta_{i}}{\beta}\left[\sqrt{\frac{b \bar{\gamma}_{1}}{\alpha \bar{\gamma}_{3 i}}} \Gamma\left(\frac{1}{2}, \frac{b \bar{\gamma}_{1}}{\alpha \bar{\gamma}_{3 i}}\right) e^{\frac{b \bar{\gamma}_{1}}{\alpha \bar{\gamma}_{3 i}}}-\sum_{j=1}^{N_{I_{2}}}\left(\frac{\bar{\gamma}_{4 j}}{b \overline{\gamma_{2}}}\right)^{N_{\mathrm{r}}} \frac{\zeta_{j} H_{1}}{\sqrt{\pi} \Gamma\left(N_{\mathrm{r}}\right)}\right],
$$

where $\Gamma(\cdot, \cdot)$ is the upper incomplete Gamma function ([31], eq.(8.350.2)) and

$$
H_{1}=H_{1,[1: 1], 0,[1: 1]}^{1,1,1,1}\left[\frac{\bar{\gamma}_{4 j}}{b \bar{\gamma}_{2}}, \frac{\alpha \bar{\gamma}_{3 i}}{b \bar{\gamma}_{1}} \mid \begin{array}{c}
\left(N_{\mathrm{r}}+1 / 2,1\right) ;\left(1-N_{\mathrm{r}}, 1\right) ;(0,1) \\
--;(0,1) ;(0,1)
\end{array}\right]
$$

with $H_{1,[1: 1], 0,[1: 1]}^{1,1,1,1}[\cdot]$ being the generalized Fox's H-function ([35], eq.(2.2.1)).

\section{Proof. See Appendix 2.}

Theorem 2 presents an analytical expression for the average SER of the system, and it applies to the generic scenario with arbitrary number of antennas, CCI, and feedback delay at any SIR. The closed-form expression involves the generalized Fox's $H$-function, which can be efficiently evaluated via the algorithm developed in ([36] Table two), hence provides a fast and reliable way to evaluate the average SER of the system.

In order to get more insights into how the system parameters such as number of antennas $N_{\mathrm{t}}$ and $N_{\mathrm{r}}$, interference, and correlation coefficient $\rho$ affect the average SER of the system, we now present simple expressions of the average SER in the high SIR regime, from which the diversity order and coding gain of the system can be easily analyzed. Similar to the outage analysis part, we consider two asymptotic SER cases depending on the value of $\rho$.

Corollary 3. With only CCI, i.e., $\rho=1$, the asymptotic SER of interference-limited dual-hop multiple antenna CSIassisted AF relaying systems is given by

$$
\bar{P}_{e}^{\infty} \approx\left\{\begin{array}{lc}
\frac{a \Gamma(k+1 / 2)}{2 b^{k} \sqrt{\pi}} \sum_{i=1}^{N_{I_{1}}} \eta_{i} k\left(\begin{array}{c}
N_{\mathrm{t}} \\
k
\end{array}\right) \sum_{n=0}^{k-1}(-1)^{n+k+1}\left(\begin{array}{c}
k-1 \\
n
\end{array}\right) \beta^{k-1} \bar{\gamma}_{3 i}^{k}\left(\frac{1}{\bar{\gamma}_{1}}\right)^{k}, & k<N_{\mathrm{r}} \\
\frac{a \Gamma(L+1 / 2)}{2 b^{L} \sqrt{\pi}} \sum_{i=1}^{N_{I_{1}}} \eta_{i} \sum_{j=1}^{N_{I_{2}}} \zeta_{j}\left[\left(\frac{\bar{\gamma}_{4 j}}{\mu}\right)^{L}-L\left(\begin{array}{c}
N_{\mathrm{t}} \\
L
\end{array}\right) \sum_{n=0}^{L-1}(-1)^{n+L}\left(\begin{array}{c}
L-1 \\
n
\end{array}\right) \beta^{L-1} \bar{\gamma}_{3 i}^{L}\right]\left(\frac{1}{\bar{\gamma}_{1}}\right)^{L}, k=N_{\mathrm{r}}=L . \\
\frac{a \Gamma\left(N_{\mathrm{r}}+1 / 2\right)}{2 b^{N_{\mathrm{r}} \sqrt{\pi}}} \sum_{j=1}^{N_{I_{2}}} \zeta_{j}\left(\frac{\bar{\gamma}_{4 j}}{\mu}\right)^{N_{\mathrm{r}}}\left(\frac{1}{\bar{\gamma}_{1}}\right)^{N_{\mathrm{r}}}, & k>N_{\mathrm{r}}
\end{array}\right.
$$

Proof. Substituting the asymptotic CDF expression presented in (22) into (26) and utilizing [31] eq.(3.351.3), the desired result can be obtained after some algebraic manipulations.

Corollary 4. With both the CCI and feedback delay, i.e., $\rho<1$, the asymptotic average SER of the interference-limited dual-hop multiple antenna CSI-assisted AF relaying systems is given by

$$
\bar{P}_{e}^{\infty} \approx\left\{\begin{array}{ll}
\frac{a}{4 b} \sum_{i=1}^{N_{I_{1}}} \eta_{i} \sum_{j=1}^{N_{I_{2}}} \zeta_{j}\left[\frac{\bar{\gamma}_{4 j}}{\mu}+k\left(\begin{array}{c}
N_{\mathrm{t}} \\
k
\end{array}\right) \sum_{n=0}^{k-1}(-1)^{n+k}\left(\begin{array}{c}
k-1 \\
n
\end{array}\right) \frac{\alpha \bar{\gamma}_{3 i}}{\beta}\right] \frac{1}{\bar{\gamma}_{1}}, N_{\mathrm{r}}=1 \\
\frac{a}{4 b} \sum_{i=1}^{N_{I_{1}}} \eta_{i} k\left(\begin{array}{c}
N_{\mathrm{t}} \\
k
\end{array}\right) \sum_{n=0}^{k-1}(-1)^{n}\left(\begin{array}{c}
k-1 \\
n
\end{array}\right) \frac{\alpha \bar{\gamma}_{3 i}}{\beta} \frac{1}{\bar{\gamma}_{1}}, & N_{\mathrm{r}}>1
\end{array} .\right.
$$

Proof. Substituting the asymptotic CDF expression presented in (23) into (26) and utilizing [31] eq.(3.351.3), the desired result can be obtained after some algebraic manipulations.

It is noted from the above two corollaries that the diversity order achieved by the system with no feedback delay is $\min \left\{k, N_{\mathrm{r}}\right\}$. However, the diversity order reduces to one when there is feedback delay. Moreover, the results suggest that the CCI only degrades the performance of the system by affecting the coding gain of the system and does not reduce the achievable diversity order. 


\subsection{Ergodic capacity}

In this section, we study the ergodic capacity of interference-limited dual-hop multiple antenna CSIassisted AF relaying systems with feedback delay. Mathematically, the ergodic capacity of the system can be expressed as

$$
C=\frac{1}{2} \mathrm{E}\left[\log _{2}(1+\gamma)\right]=\frac{1}{2} \int_{0}^{\infty} \log _{2}(1+x) f_{\gamma}(x) d x
$$

where $f_{\gamma}(x)$ denotes the PDF of the end-to-end SIR $\gamma$.

It is worth pointing out that unlike the outage probability and SER metrics which have been widely studied for various dual-hop relaying systems in the presence of CCI, few works have investigated the ergodic capacity of dualhop relaying systems with $\mathrm{CCI}$ due to the fact that it is extremely difficult to obtain tractable expressions for the ergodic capacity. Indeed, two key challenges encountered in the analysis of ergodic capacity include obtaining the exact PDF of the end-to-end SIR and taking expectation of the nonlinear $\log (\cdot)$ function. To circumvent these challenges, we hereafter seek upper and lower bounds of the ergodic capacity of interference-limited dual-hop multiple antenna CSI-assisted AF relaying systems with feedback delay. We start by presenting the following ergodic capacity upper bound.

Theorem 3. The ergodic capacity of interference-limited dual-hop multiple antenna CSI-assisted AF relaying systems with feedback delay is upper bounded by

$$
\begin{aligned}
C_{u}= & \frac{k}{2 \ln 2}\left(\begin{array}{c}
N_{\mathrm{t}} \\
k
\end{array}\right) \sum_{n=0}^{k-1} \frac{(-1)^{n}}{\beta}\left(\begin{array}{c}
k-1 \\
n
\end{array}\right) \sum_{i=1}^{N_{I_{1}}} \eta_{i 2} F_{1}\left(1,1 ; 2 ; 1-\alpha \frac{\bar{\gamma}_{3 i}}{\bar{\gamma}_{1}}\right), \\
& +\frac{1}{2 \ln 2} \sum_{j=1}^{N_{I_{2}}} \zeta_{j}\left[\frac{1}{N_{\mathrm{r}}}{ }_{2} F_{1}\left(1, N_{\mathrm{r}} ; N_{\mathrm{r}}+1 ; 1-\frac{\bar{\gamma}_{2}}{\bar{\gamma}_{4 j}}\right)-\ln \bar{\gamma}_{4 j}\right] \\
& +\frac{1}{2 \ln 2}\left\{\psi\left(N_{\mathrm{r}}\right)+\ln \bar{\gamma}_{2}+C-\ln \left(1+e^{\Lambda_{1}}+e^{\Lambda_{2}}\right)\right\},
\end{aligned}
$$

where ${ }_{2} F_{1}(\cdot, \cdot ; \cdot ; \cdot)$ is the Gauss hypergeometric function ([31], eq.(9.100)), $\psi(\cdot)$ is the Euler psi function ([31], eq.(8.360)), $C$ is the Euler constant ([31], eq.(8.367.1)), and

$$
\Lambda_{1}=k\left(\begin{array}{c}
N_{\mathrm{t}} \\
k
\end{array}\right) \sum_{n=0}^{k-1}(-1)^{n+1}\left(\begin{array}{c}
k-1 \\
n
\end{array}\right) \frac{\ln \left(\alpha / \bar{\gamma}_{1}\right)}{\beta}-\sum_{i=1}^{N_{I_{1}}} \eta_{i} \ln \bar{\gamma}_{3 i}
$$

$\Lambda_{2}=\psi\left(N_{\mathrm{r}}\right)+\ln \bar{\gamma}_{2}+C-\sum_{j=1}^{N_{I_{2}}} \zeta_{j} \ln \bar{\gamma}_{4 j}$

Proof. See Appendix 3.
Theorem 3 provides a general expression of the capacity upper bound, which is valid for arbitrary number of antenna, CCI and feedback delay. Now, we turn our attention to the ergodic capacity lower bound, and we have the following key result:

Theorem 4. The ergodic capacity of interference-limited dual-hop multiple antenna CSI-assisted AF relaying systems with feedback delay is lower bounded by

$$
\begin{aligned}
C_{l}=\frac{k}{2 \ln 2}\left(\begin{array}{c}
N_{\mathrm{t}} \\
k
\end{array}\right) \sum_{n=0}^{k-1} \frac{(-1)^{n}}{N_{\mathrm{t}}-k+n+1}\left(\begin{array}{c}
k-1 \\
n
\end{array}\right) \\
\quad \times \sum_{i=1}^{N_{I_{1}}} \eta_{i}\left[\ln \bar{\gamma}_{3 i}+{ }_{2} F_{1}\left(1,1 ; 2 ; 1-\frac{\alpha \bar{\gamma}_{3 i}}{\bar{\gamma}_{1}}\right)\right] \\
+\frac{1}{2 \ln 2}\left[\sum_{j=1}^{N_{I_{2}}} \frac{\zeta_{j}}{N_{\mathrm{r}}}{ }_{2} F_{1}\left(1, N_{\mathrm{r}} ; N_{\mathrm{r}}+1 ; 1-\frac{\bar{\gamma}_{2}}{\bar{\gamma}_{4 j}}\right)\right. \\
\left.+\psi\left(N_{\mathrm{r}}\right)+\ln \bar{\gamma}_{2}-C-\ln \left(B_{1}+B_{2}+B_{3}\right)\right],
\end{aligned}
$$

where

$$
\begin{aligned}
& B_{1}=k\left(\begin{array}{c}
N_{\mathrm{t}} \\
k
\end{array}\right) \sum_{n=0}^{k-1} \frac{(-1)^{n}}{\beta}\left(\begin{array}{c}
k-1 \\
n
\end{array}\right) \frac{\bar{\gamma}_{1}}{\alpha} \sum_{j=1}^{N_{I_{2}}} \zeta_{j} \bar{\gamma}_{4 j}, \\
& B_{2}=N_{\mathrm{r}} \bar{\gamma}_{2} \sum_{i=1}^{N_{I_{1}}} \eta_{i} \bar{\gamma}_{3 i}, \\
& B_{3}=\sum_{i=1}^{N_{I_{1}}} \eta_{i} \bar{\gamma}_{3 i} \sum_{j=1}^{N_{I_{2}}} \zeta_{j} \bar{\gamma}_{4 j} .
\end{aligned}
$$

Proof. See Appendix 4.

It is worth highlighting that the upper and lower bounds on the ergodic capacity presented in the previous theorems involve only standard functions, hence can be very fast and efficiently evaluated in popular softwares such as Matlab or Mathematica. More importantly, the derived bounds remain very tight across the entire range of SIRs as will be demonstrated in Section 5 .

\section{Optimum power allocation}

In this section, we propose optimum power allocation schemes minimizing the asymptotic outage probability with/without feedback delay. Specifically, we consider the scenario that the total transmit power between the source $S$ and the relay $R$ is fixed, i.e., $P_{s}+P_{r}=P_{t}$. 
As can be readily observed from (22), more power should be allocated to the source $S$ to suppress the interference at the relay when $k<N_{\mathrm{r}}$. In contrast, the relay node should be allocated more power to alleviate the detrimental effect of the interference at the destination when $k>N_{\mathrm{r}}$. On the other hand, the case $k=N_{\mathrm{r}}=L$ requires much more careful consideration. The optimal power allocation problem can be formulated as

$$
\begin{aligned}
& \varepsilon_{1}^{*}=\arg \min _{\varepsilon_{1}} P_{\text {out }}^{\infty}\left(\varepsilon_{1}, P_{t}\right) \\
& \text { s.t. } P_{s}+P_{r}=P_{t}, P_{s}=\varepsilon_{1} P_{t}, P_{r}=\left(1-\varepsilon_{1}\right) P_{t}, \\
& \text { and } 0<\varepsilon_{1}<1
\end{aligned}
$$

where

$$
\begin{aligned}
P_{\text {out }}^{\infty}\left(\varepsilon_{1}, P_{t}\right)= & {\left[\sum_{j=1}^{N_{I_{2}}} \zeta_{j}\left(\frac{\bar{\gamma}_{4 j}}{\lambda_{r d}\left(1-\varepsilon_{1}\right)}\right)^{L}-\sum_{i=1}^{N_{I_{1}}} \eta_{i} L\left(\begin{array}{c}
N_{\mathrm{t}} \\
L
\end{array}\right)\right.} \\
& \left.\sum_{n=0}^{L-1} \frac{(-1)^{n+L}}{\beta}\left(\begin{array}{c}
L-1 \\
n
\end{array}\right)\left(\frac{\beta \bar{\gamma}_{3 i}}{\lambda_{s r} \varepsilon_{1}}\right)^{L}\right]\left(\frac{\Delta}{P_{t}}\right)^{L} .
\end{aligned}
$$

To this end, taking the second derivative of $P_{\text {out }}^{\infty}\left(\varepsilon_{1}, P_{t}\right)$ with respect to $\varepsilon_{1}$, it can be shown that $\partial^{2} P_{\text {out }}^{\infty}\left(\varepsilon_{1}, P_{t}\right) / \partial \varepsilon_{1}^{2}$ is strictly positive in the interval $(0,1)$, which implies that the objective function is a strictly convex function of $\varepsilon_{1}$ in the interval $(0,1)$. Therefore, taking the first-order derivative of $P_{\text {out }}^{\infty}\left(\varepsilon_{1}, P_{t}\right)$ with respect to $\varepsilon_{1}$ and setting it to zero, the optimal $\varepsilon_{1}$ can be obtained. After some straightforward yet tedious algebraic manipulations, we get

$$
\varepsilon_{1}^{*}=\frac{\sqrt{L+1} A_{1} / A_{2}}{1+\sqrt{L+1} A_{1} / A_{2}},
$$

where

$$
\begin{aligned}
& A_{1}=L\left(\begin{array}{c}
N_{\mathrm{t}} \\
L
\end{array}\right) \sum_{n=0}^{L-1} \frac{(-1)^{n+L+1}}{\beta}\left(\begin{array}{c}
L-1 \\
n
\end{array}\right) \sum_{i=1}^{N_{I_{1}}} \eta_{i}\left(\frac{\beta \bar{\gamma}_{3 i}}{\lambda_{s r}}\right)^{L} \text { and } \\
& A_{2}=\sum_{j=1}^{N_{I_{2}}} \zeta_{j}\left(\frac{\bar{\gamma}_{4 j}}{\lambda_{r d}}\right)^{L} .
\end{aligned}
$$

Similarly, for the case $\rho<1$, more power should be allocated to the source node when the destination node has more than a single antenna. On the other hand, when the destination is equipped with a single antenna, the optimal power allocation problem can be formulated as

$$
\begin{aligned}
& \varepsilon_{2}^{*}=\arg \min _{\varepsilon_{2}} P_{\text {out }}^{\infty}\left(\varepsilon_{2}, P_{t}\right) \\
& \text { s.t. } P_{s}+P_{r}=P_{t}, P_{s}=\varepsilon_{2} P_{t}, P_{r}=\left(1-\varepsilon_{2}\right) P_{t} \text {, } \\
& \text { and } 0<\varepsilon_{2}<1,
\end{aligned}
$$

where

$$
\begin{aligned}
P_{\text {out }}^{\infty}\left(\varepsilon_{2}, P_{t}\right)= & {\left[\sum_{j=1}^{N_{I_{2}}} \frac{\zeta_{j} \bar{\gamma}_{4 j}}{\lambda_{r d}\left(1-\varepsilon_{2}\right)}+\sum_{i=1}^{N_{I_{1}}} \eta_{i} k\left(\begin{array}{c}
N_{\mathrm{t}} \\
k
\end{array}\right)\right.} \\
& \left.\sum_{n=0}^{k-1} \frac{(-1)^{n}}{\beta}\left(\begin{array}{c}
k-1 \\
n
\end{array}\right) \frac{\alpha \bar{\gamma}_{3 i}}{\lambda_{s r} \varepsilon_{2}}\right] \frac{\Delta}{P_{t}} .
\end{aligned}
$$

After some algebraic manipulations, it can be proven that the second derivative of $P_{\text {out }}^{\infty}\left(\varepsilon_{2}, P_{t}\right)$ with respect to $\varepsilon_{2}$ is strictly positive, which implies that the objective function is a strictly convex function of $\varepsilon_{2}$. Setting the first derivative of the objective function with respect to $\varepsilon_{2}$ to zero, the optimal power allocation factor can be derived as

$$
\varepsilon_{2}^{*}=\frac{\sqrt{B_{1} / B_{2}}}{1+\sqrt{B_{1} / B_{2}}},
$$

where

$$
\begin{aligned}
B_{1} & =k\left(\begin{array}{c}
N_{\mathrm{t}} \\
k
\end{array}\right) \sum_{n=0}^{k-1}(-1)^{n} \frac{\alpha}{\beta}\left(\begin{array}{c}
k-1 \\
n
\end{array}\right) \sum_{i=1}^{N_{I_{1}}} \eta_{i} \frac{\bar{\gamma}_{3 i}}{\lambda_{s r}} \text { and } \\
B_{2} & =\sum_{j=1}^{N_{I_{2}}} \zeta_{j} \frac{\bar{\gamma}_{4 j}}{\lambda_{r d}}
\end{aligned}
$$

\section{Numerical results}

In this section, we perform extensive numerical simulations to validate analytical results developed in the previous sections and to investigate the impact of number of antennas, CCI, and feedback delay on the performance of interference-limited dual-hop multiple antenna CSIassisted AF relaying systems with feedback delay. In all simulations, unless otherwise specified, we use the following set of parameters: $P_{r}=P_{s}, \lambda_{s r}=1$, and $\lambda_{r d}=1$. The rate threshold $R$ is set $5 \mathrm{~W}$, and the BPSK modulation, i.e., $a=1$ and $b=1$, is used.

Figure 2 plots the outage probability of interferencelimited dual-hop multiple antenna CSI-assisted AF relaying systems with different $\rho$. As shown in the figure, the analytical results, i.e., the outage lower bounds, are sufficiently tight across the entire SIR range of interest, while the high SIR approximations work quite well even at moderate SIRs (i.e., $\bar{\gamma}_{1}=20 \mathrm{~W}$ ). We also observe that the outage performance is degraded when the feedback delay becomes large, i.e., $\rho$ gets smaller. Moreover, the full diversity order can be achieved without feedback delay, i.e., $\rho=1$. However, only unity diversity order is achieved for the case of feedback delay, and increasing $k$ improves the outage probability by affecting the coding gain. On the other hand, Figure 3 illustrates the impact of number of interference on the outage performance under two cases: $N_{I_{1}}=N_{I_{2}}=2$ and $N_{I_{1}}=N_{I_{2}}=4$, respectively. It is worth 


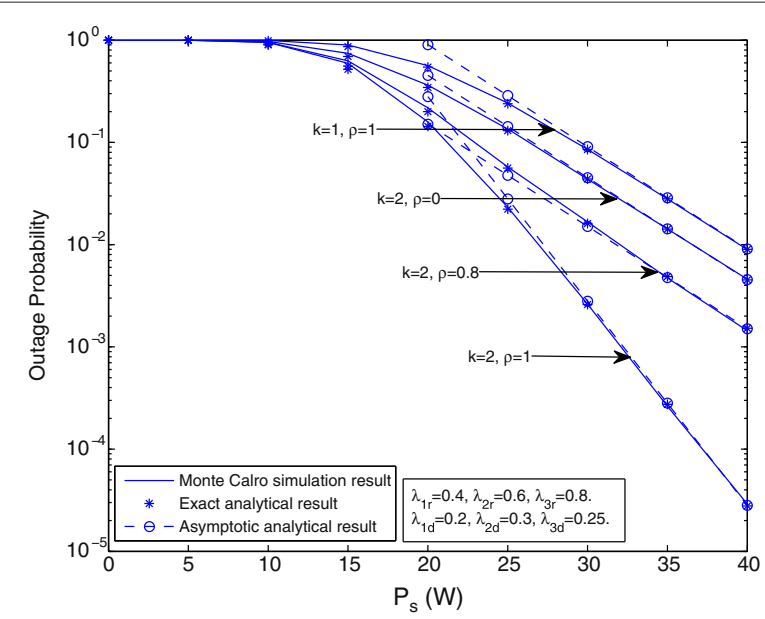

Figure 2 Outage probability versus the transmit power $P_{s}$ when $N_{\mathrm{t}}=N_{\mathrm{r}}=2, P_{i r}=5 \mathrm{~W}, P_{j d}=0 \mathrm{~W}$, and $N_{I_{1}}=N_{I_{2}}=3$.

noting that increasing the number of interferers results in a higher outage probability.

Figure 4 examines the average SER of interferencelimited dual-hop multiple antenna CSI-assisted AF relaying systems with different $\rho$. As can be clearly seen from the figure, the analytical results are in close agreement with the Monte Carlo simulations at the entire SIR region, while high SIR curves are also compatible with the simulations. Specifically, when the SIR is in the high regime, the theoretical lines agree with the simulations, which verifies the correctness of the upper bound on the SIR. Furthermore, in the case of perfect feedback (no feedback delay), the diversity order of the select order $k$ is achieved. However, due to the feedback delay in selecting the antenna, the diversity order is degraded to one regardless of the

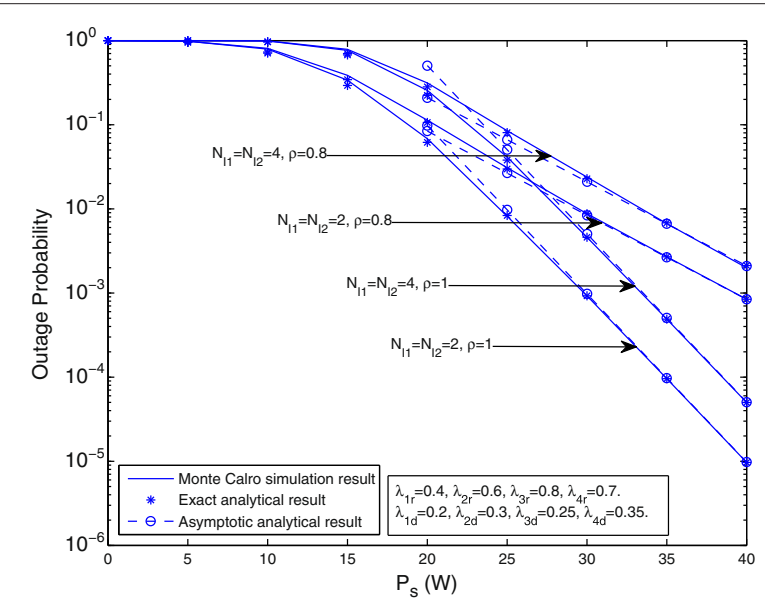

Figure 3 Outage probability versus the transmit power $\boldsymbol{P}_{s}$ under different interference when $P_{i r}=5 \mathrm{~W}, P_{j d}=0 \mathrm{~W}, N_{\mathrm{t}}=N_{\mathrm{r}}=2$, and $k=2$.

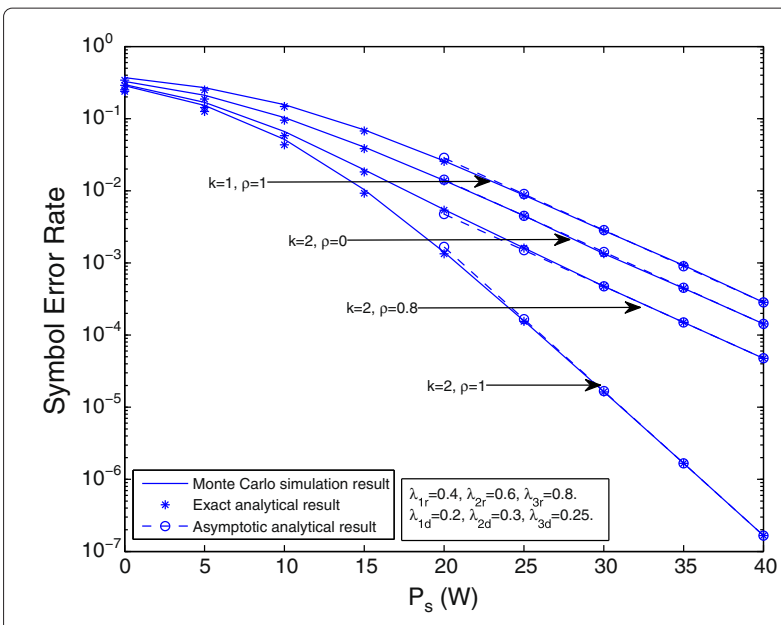

Figure 4 Symbol error rate versus the transmit power $P_{s}$ when $N_{\mathrm{t}}=N_{\mathrm{r}}=2, P_{i r}=5 \mathrm{~W}, P_{j d}=0 \mathrm{~W}$, and $N_{I_{1}}=N_{I_{2}}=3$.

number of antennas. Further, we can see that when $\rho$ increases, the average SER is improved. This can be clearly noticed in the two curves of $\rho=0$ and 0.8 in the figure. Besides, Figure 5 shows the relation between the average SER and the number of interference, and we adopt the same setting parameters as Figure 3. From the figure, more interference significantly degrades the average SER performance due to the increasing interference power.

Figure 6 plots the proposed upper and lower bounds for ergodic capacity of interference-limited dual-hop multiple antenna CSI-assisted AF relaying systems with different $\rho$. As can be readily seen, the derived upper and lower bounds keep tight across the entire SIR range. We also observe the intuitive result that the ergodic capacity becomes less as $\rho$ gets smaller (i.e., the feedback delay

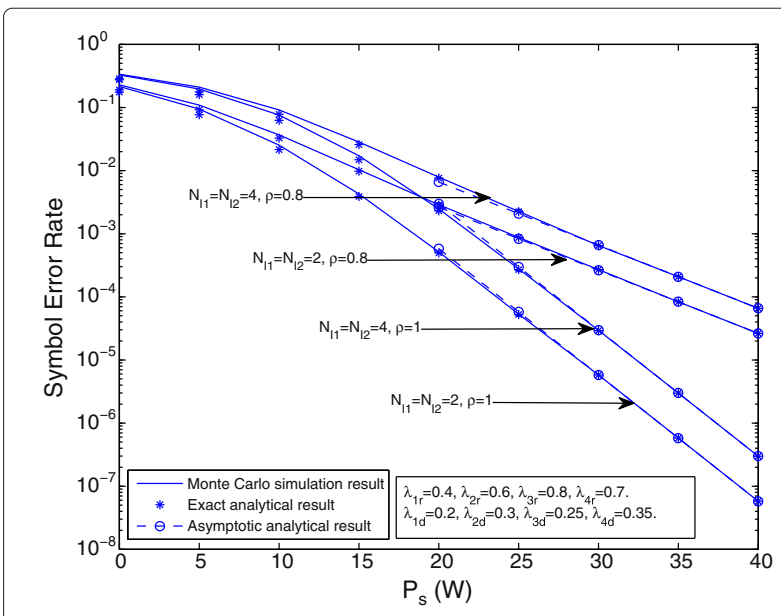

Figure 5 Symbol error rate versus the transmit power $P_{s}$ under different interference when $P_{i r}=5 \mathrm{~W}, P_{j d}=0 \mathrm{~W}, N_{\mathrm{t}}=N_{\mathrm{r}}=2$, and $k=2$. 


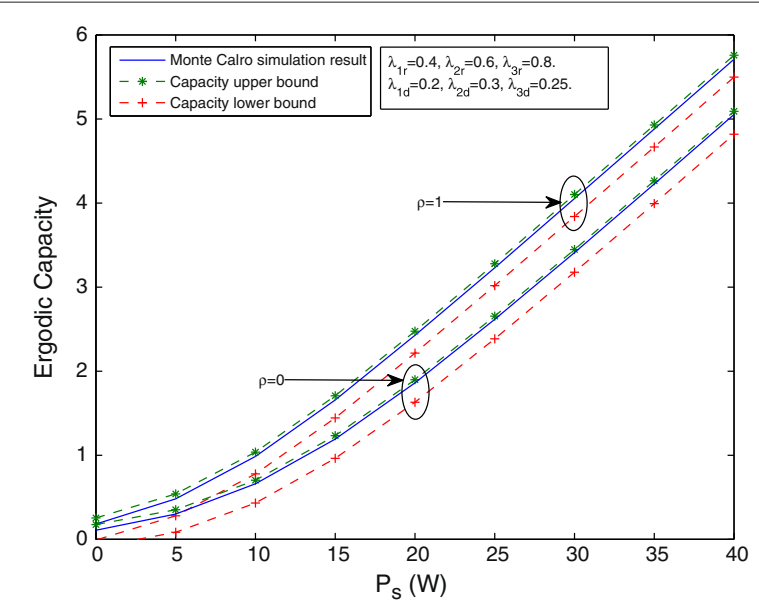

Figure 6 Ergodic capacity versus the transmit power $\boldsymbol{P}_{\boldsymbol{s}}$ when $N_{\mathrm{t}}=N_{\mathrm{r}}=3, k=3, P_{i r}=5 \mathrm{~W}, P_{j d}=0 \mathrm{~W}$, and $N_{I_{1}}=N_{I_{2}}=3$.

becomes large). Furthermore, we characterize the number of interference on the ergodic capacity in Figure 7. It is shown that more interference results in the lower ergodic capacity under different cases.

Figure 8 provides the outage performance comparison between the optimal power allocation scheme and the equal power allocation scheme with/without feedback delay, respectively. We can see that the outage probability of the optimal power allocation scheme outperforms that of the equal power allocation scheme. This is due to the fact that more power will be allocated to the link which is subjected to more interference power to overcome the interference. Furthermore, it is worth noting that the optimal power allocation scheme can further bring the coding gain compared with the equal power allocation scheme to the underlying system.

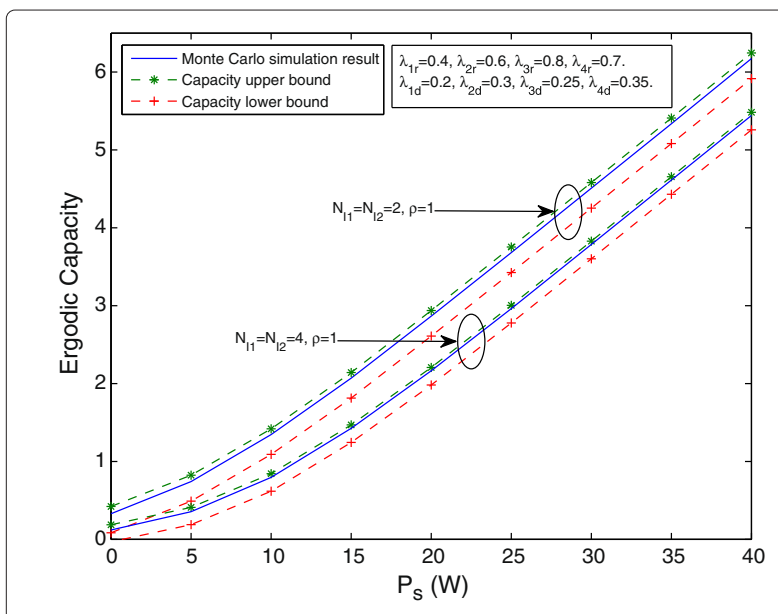

Figure 7 Ergodic capacity versus the transmit power $\boldsymbol{P}_{s}$ under different interference when $N_{\mathrm{t}}=N_{\mathrm{r}}=3, k=3, P_{i r}=5 \mathrm{~W}$, and $P_{j d}=0 \mathrm{~W}$.

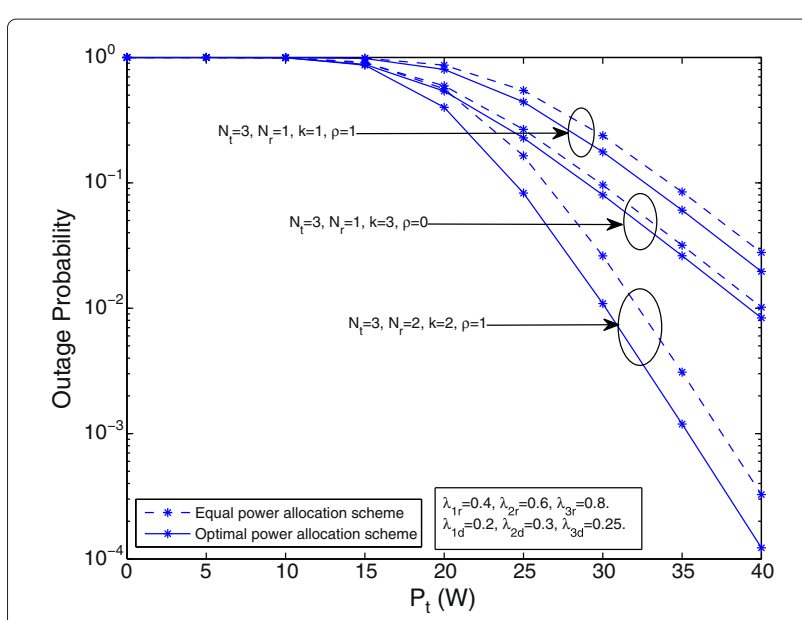

Figure 8 Outage probability comparison of two power allocation schemes when $P_{i r}=5 \mathrm{~W}, P_{j d}=0 \mathrm{~W}$, and $N_{I_{1}}=N_{I_{2}}=3$.

\section{Conclusions}

In this paper, we have investigated the impact of number of antennas, CCI, and feedback delay on the performance of interference-limited dual-hop multiple antenna CSIassisted AF relaying networks with arbitrary TAS/MRC. Specifically, based on a tight upper bound for the effective end-to-end SIR, the closed-form approximate expressions for the outage probability and the average SER were derived, which provided a fast and efficient tool for evaluating the impact of the key parameters on the system performance. Simulation results illustrated that the derived approximations for the outage probability and the average SER achieved a good match with the exact results. Furthermore, simple and high informative expressions were also provided to obtain the diversity order and the coding gain of the system with perfect feedback and delayed feedback. The finding of the paper suggested that the full diversity order of $\min \left\{k, N_{\mathrm{r}}\right\}$ was achieved under perfect feedback, whereas the diversity order was degraded to one underdelayed feedback. More importantly, some new analytical upper and lower bounds were first derived for the ergodic capacity of the considered system, which demonstrated that the proposed bounds worked quite well under different cases. In addition, the optimal power allocation between the source and the relay was proposed to minimize the asymptotic outage probability. A profound reduction in the outage probability was attained using the optimal power allocation compared to the equal power allocation due to additional coding gain.

\section{Endnote}

${ }^{\mathrm{a}}$ The reason for using the $k$ th worst antenna selection rather than the best antenna selection has been explained in $[37,38]$. 


\section{Appendices}

\section{Appendix 1}

Utilizing the McLaurin series expansion for the exponential function in (11) and performing some mathematical manipulations, the asymptotic CDF of $\tilde{\gamma}_{1(k)} / \gamma_{I_{1}}$ can be derived as

$$
\begin{aligned}
F_{\tilde{\gamma}_{1(k)} / \gamma_{I_{1}}}(x) \stackrel{\bar{\gamma}_{1} \rightarrow \infty}{\approx} \sum_{i=1}^{N_{I_{1}}} \eta_{i} k\left(\begin{array}{c}
N_{\mathrm{t}} \\
k
\end{array}\right) \sum_{n=0}^{k-1}(-1)^{n+k+1} \\
\times\left(\begin{array}{c}
k-1 \\
n
\end{array}\right) \beta^{k-1}\left(\frac{x \bar{\gamma}_{3 i}}{\bar{\gamma}_{1}}\right)^{k},
\end{aligned}
$$

where $\beta=N_{\mathrm{t}}-k+n+1$.

In order to get the asymptotic CDF of $\gamma_{2} / \gamma_{I_{2}}$, we first express the incomplete Gamma function using the series expansion as ([31], eq.(8.354.1))

$$
\gamma(m, \theta x)=(\theta x)^{m} \sum_{n=0}^{\infty} \frac{(-1)^{n}(\theta x)^{n}}{n !(m+n)} \stackrel{x \rightarrow 0}{\approx} \frac{(\theta x)^{m}}{m}
$$

Then, substituting (48) into (20) and carrying out the integration with the help of [31] eq.(3.351.3), the asymptotic CDF of $\gamma_{2} / \gamma_{I_{2}}$ can be computed as

$$
F_{\gamma_{2} / \gamma_{2}}(x)^{\bar{\gamma}_{2} \rightarrow \infty} \sum_{j=1}^{N_{I_{2}}} \zeta_{j}\left(\frac{x \bar{\gamma}_{4 j}}{\bar{\gamma}_{2}}\right)^{N_{\mathrm{r}}}
$$

Finally, substituting (47) and (49) into (14) and simplifying them, the asymptotic outage probability is derived as

$$
\begin{aligned}
P_{\text {out }}^{\infty}(z) \approx 1- & \sum_{i=1}^{N_{I_{1}}} \eta_{i}\left[1+k\left(\begin{array}{c}
N_{\mathrm{t}} \\
k
\end{array}\right) \sum_{n=0}^{k-1}(-1)^{n+k}\right. \\
& \left.\times\left(\begin{array}{c}
k-1 \\
n
\end{array}\right) \beta^{k-1}\left(\bar{\gamma}_{3 i} z\right)^{k}\right] \\
& \times \sum_{j=1}^{N_{I_{2}}} \zeta_{j}\left[1-\left(\frac{\bar{\gamma}_{4 j}}{\mu} z\right)^{N_{\mathrm{r}}}\right] .
\end{aligned}
$$

To this end, the desired result can be obtained after some mathematical manipulations.

\section{Appendix 2}

Substituting (13) into (26), the average SER can be expressed as

$$
\begin{aligned}
\bar{P}_{e} \approx & \frac{a}{2} \sqrt{\frac{b}{\pi}} \underbrace{\int_{0}^{\infty} \frac{e^{-b x}}{\sqrt{x}} d x}_{I_{1}}-\frac{a}{2} \sqrt{\frac{b}{\pi}} k\left(\begin{array}{c}
N_{\mathrm{t}} \\
k
\end{array}\right) \\
& \times \sum_{n=0}^{k-1} \frac{(-1)^{n}}{N_{\mathrm{t}}-k+n+1}\left(\begin{array}{c}
k-1 \\
n
\end{array}\right) \sum_{i=1}^{N_{I_{1}}} \eta_{i} \bar{\gamma}_{1} \\
& \times[\underbrace{\int_{0}^{\infty} \frac{e^{-b x} x^{-1 / 2}}{\alpha \bar{\gamma}_{3 i}\left(x+\bar{\gamma}_{1} / \alpha \bar{\gamma}_{3 i}\right)} d x}_{I_{2}}-\sum_{i=1}^{N_{I_{2}}} \frac{\zeta_{j}}{\bar{\gamma}_{1}}\left(\frac{\bar{\gamma}_{4 j}}{\bar{\gamma}_{2}}\right)^{N_{\mathrm{r}}} \\
& \times \underbrace{\int_{0}^{\infty} e^{-b x} x^{N_{\mathrm{r}}-\frac{1}{2}}\left(1+\frac{\alpha \bar{\gamma}_{3 i} x}{\bar{\gamma}_{1}}\right)^{-1}\left(1+\frac{\bar{\gamma}_{4 j} x}{\bar{\gamma}_{2}}\right)^{-N_{\mathrm{r}}} d x}_{I_{3}}] .
\end{aligned}
$$

With the help of [31] eq.(3.361.2), the integral $I_{1}$ can be computed as

$$
I_{1}=\sqrt{\frac{\pi}{b}} .
$$

Also, utilizing [31] eq.(3.383.10), the integral $I_{2}$ can be evaluated as

$$
I_{2}=\sqrt{\frac{\pi}{\alpha \bar{\gamma}_{3 i} \bar{\gamma}_{1}}} e^{\frac{b \bar{\gamma}_{1}}{\alpha \bar{\gamma}_{3 i}}} \Gamma\left(\frac{1}{2}, \frac{b \bar{\gamma}_{1}}{\alpha \bar{\gamma}_{3 i}}\right),
$$

where $\Gamma(\cdot, \cdot)$ is the upper incomplete Gamma function [28] eq.(8.350.2). To evaluate the integral $I_{3}$, we first exploit the equalities ([39], eq.(8.4.23.3)) as follows:

$$
\begin{aligned}
& \left(1+\frac{\alpha \bar{\gamma}_{3 i}}{\bar{\gamma}_{1}} x\right)^{-1}=H_{1,1}^{1,1}\left[\left.\frac{\alpha \bar{\gamma}_{3 i}}{\bar{\gamma}_{1}} x\right|_{(0,1)} ^{(0,1)}\right] \\
& \left(1+\frac{\bar{\gamma}_{4 j}}{\bar{\gamma}_{2}} x\right)^{-N_{\mathrm{r}}}=H_{1,1}^{1,1}\left[\left.\frac{\bar{\gamma}_{4 j}}{\bar{\gamma}_{2}} x\right|_{(0,1)} ^{\left(1-N_{\mathrm{r}}, 1\right)}\right] /\left(N_{\mathrm{r}}-1\right) !
\end{aligned}
$$

where $H_{c, d}^{a, b}[\cdot]$ is the Fox's $H$-function ([39], eq.(8.3.1.1)). To this end, with the help of [35] eq.(2.6.2), $I_{3}$ can be derived as

$$
\begin{aligned}
I_{3}= & \frac{1}{b^{N_{\mathrm{r}}+1 / 2} \Gamma\left(N_{\mathrm{r}}\right)} H_{1,[1: 1], 0,[1: 1]}^{1,1,1,1,1} \\
& \times\left[\frac{\bar{\gamma}_{4 j}}{b \bar{\gamma}_{2}}, \frac{\alpha \bar{\gamma}_{3 i}}{b \bar{\gamma}_{1}} \mid \begin{array}{c}
\left(N_{\mathrm{r}}+1 / 2,1\right) ;\left(1-N_{\mathrm{r}}, 1\right) ;(0,1) \\
--;(0,1) ;(0,1)
\end{array}\right],
\end{aligned}
$$

where $H_{1,[1: 1], 0,[1: 1]}^{1,1,1,1,1}[\cdot]$ is the generalized Fox's $H$-function ([35], eq.(2.2.1)). Finally, substituting the integrals $I_{1}, I_{2}$, and $I_{3}$ into (51) yields the final result given in (27). 


\section{Appendix 3}

Capitalizing on the techniques proposed in recent works $[40,41]$, we get the following upper bound for the ergodic capacity of the system.

$$
\begin{aligned}
C_{u}= & \frac{1}{2} \mathrm{E}\left[\log _{2}\left(1+\frac{\tilde{\gamma}_{1(k)}}{\gamma_{I_{1}}}\right)\right]+\frac{1}{2} \mathrm{E}\left[\log _{2}\left(1+\frac{\gamma_{2}}{\gamma_{I_{2}}}\right)\right] \\
& -\frac{1}{2} \log _{2}\left\{1+e^{\mathrm{E}\left[\ln \left(\tilde{\gamma}_{1(k)} / \gamma_{I_{1}}\right)\right]}+e^{\mathrm{E}\left[\ln \left(\gamma_{2} / \gamma_{I_{2}}\right)\right]}\right\} .
\end{aligned}
$$

We start with the first term in (57). Noticing that the random variables $\tilde{\gamma}_{1(k)}$ and $\gamma_{I_{1}}$ are statistically independent, $\mathrm{E}\left[\log _{2}\left(1+\frac{\widetilde{\gamma}_{1(k)}}{\gamma_{I_{1}}}\right)\right]$ can be expanded as

$$
\begin{aligned}
\mathrm{E} & {\left[\log _{2}\left(1+\frac{\tilde{\gamma}_{1(k)}}{\gamma_{I_{1}}}\right)\right] } \\
& =\frac{1}{\ln 2} \underbrace{\int_{0}^{\infty} \int_{0}^{\infty} \ln (x+y) f_{\widetilde{\gamma}_{1(k)}}(x) d x f_{\gamma_{I_{1}}}(y) d y}_{I_{4}} \\
& -\frac{1}{\ln 2} \underbrace{\int_{0}^{\infty} \ln y f_{\gamma_{I_{1}}}(y) d y}_{I_{5}} .
\end{aligned}
$$

Substituting (10) and (15) into (58), the integral $I_{4}$ can be simplified as

$$
\begin{aligned}
I_{4}= & k\left(\begin{array}{c}
N_{\mathrm{t}} \\
k
\end{array}\right) \sum_{n=0}^{k-1} \frac{(-1)^{n}}{\bar{\gamma}_{1}}\left(\begin{array}{c}
k-1 \\
n
\end{array}\right) \frac{\alpha}{N_{\mathrm{t}}-k+n+1} \\
& \times \sum_{i=1}^{N_{I_{1}}} \frac{\eta_{i}}{\bar{\gamma}_{3 i}} \int_{0}^{\infty} \int_{0}^{\infty} \ln (x+y) e^{-\frac{\alpha}{\bar{\gamma}_{1}} x} d x e^{-\frac{y}{\bar{\gamma}_{3 i}}} d y .
\end{aligned}
$$

To this end, utilizing [31] eq.(4.337.1) and [31] eq.(6.228.2), we get

$$
\begin{aligned}
I_{4}= & k\left(\begin{array}{c}
N_{\mathrm{t}} \\
k
\end{array}\right) \sum_{n=0}^{k-1} \frac{(-1)^{n}}{N_{\mathrm{t}}-k+n+1}\left(\begin{array}{c}
k-1 \\
n
\end{array}\right) \\
& \times \sum_{i=1}^{N_{I_{1}}} \eta_{i}\left[\ln \bar{\gamma}_{3 i}+{ }_{2} F_{1}\left(1,1 ; 2 ; 1-\alpha \frac{\bar{\gamma}_{3 i}}{\bar{\gamma}_{1}}\right)-C\right],
\end{aligned}
$$

where ${ }_{2} F_{1}(\cdot, \cdot ; \cdot ; \cdot)$ is the Gauss hypergeometric function ([31], eq.(9.100)), and $C$ is the Euler constant ([31], eq. (8.367.1)).

On the other hand, by using the PDF of $\gamma_{I_{1}}$ and with the help of [31] eq.(4.331.1), the integral $I_{5}$ can be easily computed as

$$
I_{5}=\sum_{i=1}^{N_{I_{1}}} \eta_{i}\left(\ln \bar{\gamma}_{3 i}-C\right)
$$

Similarly, due to the independence of $\gamma_{2}$ and $\gamma_{I_{2}}$, the second term $E\left[\log _{2}\left(1+\frac{\gamma_{2}}{\gamma_{I_{2}}}\right)\right]$ in (57) can be expressed as

$$
\begin{aligned}
\mathrm{E}\left[\log _{2}\left(1+\frac{\gamma_{2}}{\gamma_{I_{2}}}\right)\right] \\
=\frac{1}{\ln 2} \underbrace{\int_{0}^{\infty} \int_{0}^{\infty} \ln (x+y) f_{\gamma_{2}}(x) d x f_{\gamma_{I_{2}}}(y) d y}_{I_{6}} \\
\quad-\frac{1}{\ln 2} \underbrace{\int_{0}^{\infty} \ln y f_{\gamma_{I_{2}}}(y) d y}_{I_{7}} .
\end{aligned}
$$

Utilizing the PDF of $f_{\gamma_{2}}(x)$ and $f_{\gamma_{I_{2}}}(y)$, and exploiting [31] eq.(4.337.1), we have

$$
\begin{aligned}
I_{6}= & \frac{1}{\left(N_{\mathrm{r}}-1\right) ! \bar{\gamma}_{2}^{N_{\mathrm{r}}}} \sum_{j=1}^{N_{I_{2}}} \zeta_{j}[\underbrace{\int_{0}^{\infty} x^{N_{\mathrm{r}}-1} e^{-\frac{x}{\bar{\gamma}_{2}}} \ln x d x}_{I_{8}} \\
& -\underbrace{\int_{0}^{\infty} x^{N_{\mathrm{r}}-1} \mathrm{Ei}\left(-\frac{x}{\bar{\gamma}_{4 j}}\right) e^{-x\left(\frac{1}{\bar{\gamma}_{2}}-\frac{1}{\bar{\gamma}_{4 j}}\right)} d x}_{I_{9}}]
\end{aligned}
$$

where $\mathrm{Ei}(\cdot)$ is the exponential integral function ([31], eq.(8.211.1)). Now, $I_{8}$ can be evaluated with the help of [31] eq.(4.352.1) as

$$
I_{8}=\bar{\gamma}_{2}^{N_{\mathrm{r}}}\left(N_{\mathrm{r}}-1\right) !\left[\psi\left(N_{\mathrm{r}}\right)+\ln \bar{\gamma}_{2}\right]
$$

where $\psi(\cdot)$ is the Euler psi function ([31], eq.(8.360)), while $I_{9}$ can be computed with the help of [31] eq.(6.228.2) as

$$
I_{9}=-\frac{\bar{\gamma}_{2}^{N_{\mathrm{r}}}\left(N_{\mathrm{r}}-1\right) !}{N_{\mathrm{r}}}{ }_{2} F_{1}\left(1, N_{\mathrm{r}} ; N_{\mathrm{r}}+1 ; 1-\frac{\bar{\gamma}_{2}}{\bar{\gamma}_{4 j}}\right)
$$

On the other hand, the integral $I_{7}$ can be computed via ([31], eq. (4.331.1))

$$
I_{7}=\sum_{j=1}^{N_{I_{2}}} \zeta_{j}\left(\ln \bar{\gamma}_{4 j}-C\right)
$$

Now, we turn our attention to compute $\mathrm{E}\left[\ln \left(\tilde{\gamma}_{1(k)} / \gamma_{I_{1}}\right)\right]$ and $E\left[\ln \left(\gamma_{2} / \gamma_{I_{2}}\right)\right]$. Using the PDFs of $\tilde{\gamma}_{1(k)}$ and $\gamma_{I_{1}}$, the 
first term can be evaluated after some simple manipulations with the aid of [31] eq.(4.331.1) as

$$
\begin{aligned}
\mathrm{E}\left[\ln \left(\widetilde{\gamma}_{1(k)} / \gamma_{I_{1}}\right)\right]= & k\left(\begin{array}{c}
N_{\mathrm{t}} \\
k
\end{array}\right) \sum_{n=0}^{k-1}(-1)^{n+1}\left(\begin{array}{c}
k-1 \\
n
\end{array}\right) \\
& \times \frac{\ln \left(\alpha / \bar{\gamma}_{1}\right)}{N_{\mathrm{t}}-k+n+1}-\sum_{i=1}^{N_{I_{1}}} \eta_{i} \ln \bar{\gamma}_{3 i} .
\end{aligned}
$$

Performing the required integration with the help of [31] eqs.(4.352.1) and (4.331.1), the second term can be evaluated as

$$
\mathrm{E}\left[\ln \left(\gamma_{2} / \gamma_{I_{2}}\right)\right]=\psi\left(N_{\mathrm{r}}\right)+\ln \bar{\gamma}_{2}+\boldsymbol{C}-\sum_{j=1}^{N_{I_{2}}} \zeta_{j} \ln \bar{\gamma}_{4 j} .
$$

To this end, pulling everything together, we obtain the desired result.

\section{Appendix 4}

Substituting (4) into (31) and performing some simple algebraic manipulations, the ergodic capacity can be written as

$$
\begin{aligned}
C= & \frac{1}{2} \mathrm{E}\left[\log _{2}\left(\tilde{\gamma}_{1(k)}+\gamma_{I_{1}}\right)\right]+\frac{1}{2} \mathrm{E}\left[\log _{2}\left(\gamma_{2}+\gamma_{I_{2}}\right)\right] \\
& -\frac{1}{2} \mathrm{E}\left[\log _{2}\left(\widetilde{\gamma}_{1(k)} \gamma_{I_{2}}+\gamma_{2} \gamma_{I_{2}}+\gamma_{I_{1}} \gamma_{I_{2}}\right)\right] .
\end{aligned}
$$

Applying Jensen's inequality on the third term, we obtain the following capacity lower bound:

$$
\begin{aligned}
C_{l}= & \frac{1}{2} \mathrm{E}\left[\log _{2}\left(\widetilde{\gamma}_{1(k)}+\gamma_{I_{1}}\right)\right]+\frac{1}{2} \mathrm{E}\left[\log _{2}\left(\gamma_{2}+\gamma_{I_{2}}\right)\right] \\
& -\frac{1}{2} \log _{2}\left[\mathrm{E}\left(\widetilde{\gamma}_{1(k)} \gamma_{I_{2}}\right)+\mathrm{E}\left(\gamma_{2} \gamma_{I_{2}}\right)+\mathrm{E}\left(\gamma_{I_{1}} \gamma_{I_{2}}\right)\right] .
\end{aligned}
$$

From $I_{4}$ and $I_{6}$, the first two terms of (70) can be computed in closed-form as

$$
\begin{aligned}
\mathrm{E}\left[\log _{2}\left(\widetilde{\gamma}_{1(k)}+\gamma_{I_{1}}\right)\right] \\
=\frac{k}{\ln 2}\left(\begin{array}{c}
N_{\mathrm{t}} \\
k
\end{array}\right) \sum_{n=0}^{k-1} \frac{(-1)^{n}}{N_{\mathrm{t}}-k+n+1}\left(\begin{array}{c}
k-1 \\
n
\end{array}\right) \sum_{i=1}^{N_{I_{1}}} \eta_{i} \ln \bar{\gamma}_{3 i} \\
\quad+\frac{k}{\ln 2}\left(\begin{array}{c}
N_{\mathrm{t}} \\
k
\end{array}\right) \sum_{n=0}^{k-1} \frac{(-1)^{n}}{N-k+n+1}\left(\begin{array}{c}
k-1 \\
n
\end{array}\right) \\
\quad \times \sum_{i=1}^{N_{I_{1}}} \eta_{i 2} F_{1}\left(1,1 ; 2 ; 1-\frac{\alpha \bar{\gamma}_{3 i}}{\bar{\gamma}_{1}}\right)-\frac{C}{\ln 2}
\end{aligned}
$$

$$
\begin{aligned}
\mathrm{E}\left[\log _{2}\left(\gamma_{2}+\gamma_{I_{2}}\right)\right]= & \frac{1}{\ln 2} \sum_{j=1}^{N_{I_{2}}} \frac{\zeta_{j}}{N_{\mathrm{r}}}{ }_{2} F_{1}\left(1, N_{\mathrm{r}} ; N_{\mathrm{r}}+1 ; 1-\frac{\bar{\gamma}_{2}}{\bar{\gamma}_{4 j}}\right) \\
& +\frac{\psi\left(N_{\mathrm{r}}\right)+\ln \bar{\gamma}_{2}}{\ln 2} .
\end{aligned}
$$

Now, we look at the third term. Since the other two expectations have the same integral forms as the expectation $\mathrm{E}\left(\tilde{\gamma}_{1(k)} \gamma_{I_{2}}\right)$, we focus on analyzing the expectation $\mathrm{E}\left(\widetilde{\gamma}_{1(k)} \gamma_{I_{2}}\right)$. Due to the independence of $\widetilde{\gamma}_{1(k)}$ and $\gamma_{I_{2}}$, the first expectation simplifies to

$$
\mathrm{E}\left(\tilde{\gamma}_{1(k)} \gamma_{I_{2}}\right)=k\left(\begin{array}{c}
N_{\mathrm{t}} \\
k
\end{array}\right) \sum_{n=0}^{k-1} \frac{(-1)^{n}}{N_{\mathrm{t}}-k+n+1}\left(\begin{array}{c}
k-1 \\
n
\end{array}\right) \frac{\bar{\gamma}_{1}}{\alpha} \sum_{j=1}^{N_{I_{2}}} \zeta_{j} \bar{\gamma}_{4 j},
$$

where we have used [31] eq.(3.351.3) to solve the corresponding integral.

Similarly, the other two expectations can be derived as

$$
\begin{aligned}
& \mathrm{E}\left(\gamma_{2} \gamma_{I_{1}}\right)=N_{\mathrm{r}} \bar{\gamma}_{2} \sum_{i=1}^{N_{I_{1}}} \eta_{i} \bar{\gamma}_{3 i}, \\
& \mathrm{E}\left(\gamma_{I_{1}} \gamma_{I_{2}}\right)=\sum_{i=1}^{N_{I_{1}}} \eta_{i} \bar{\gamma}_{3 i} \sum_{j=1}^{N_{I_{2}}} \zeta_{j} \bar{\gamma}_{4 j} .
\end{aligned}
$$

To this end, pulling everything together yields the desired result.

\section{Competing interests}

The authors declare that they have no competing interests.

\section{Authors' contributions}

JW carried out the design of the framework, participated in the sequence alignment and drafted the manuscript. YH participated in the design of the system model and performed the mathematical analysis. CZ revised the manuscript, and participated in its design and coordination. FAQ revised the manuscript, and done the simulation results. QW participated in the sequence alignment. YC checked the manuscript. All authors read and approved the final manuscript.

\section{Acknowledgements}

This work was supported by the National Science Foundation of China (grants numbers 61201229, 61172062, and 61072044), the Jiangsu Provincial Natural Science Foundation of China (grant number BK2011116), the Zhejiang Provincial Natural Science Foundation of China (grant number LQ12F01006), the Fundamental Research Funds for Central Universities (2012QNA5011), the Open Research Fund of National Mobile Communications Research Laboratory, Southeast University (grant number 2013D06), and the Qatar National Research Fund (JSREP grant number 3-039-2-010).

\section{Author details}

${ }^{1}$ College of Communications Engineering, PLA University of Science and Technology, Nanjing, 210007, China. ${ }^{2}$ Institute of Information and Communication Engineering, Zhejiang University, Hangzhou, 310014, China. ${ }^{3}$ The National Mobile Communications Research Laboratory, Southeast University, Nanjing, 210018, China. ${ }^{4}$ Electrical and Computer Engineering Program, Texas A\&M University at Qatar, P.O. Box 23874, Doha, Qatar. 


\section{References}

1. JN Laneman, DNC Tse, GW Wornell, Cooperative diversity in wireless networks: efficient protocols and outage behavior. IEEE Trans. Inf. Theory. 50(12), 3062-3080 (2004)

2. MO Hasna, M-S Alouini, A performance study of dual-hop transmissions with fixed gain relays. IEEE Trans. Wireless Commun. 3(6), 1963-1964 (2004)

3. MO Hasna, MS Alouini, End-to-end performance of transmission systems with relays over Rayleigh-fading channels. IEEE Trans. Wireless Commun. 2(6), 1126-1131 (2003)

4. DB da Costa, S Aïssa, Cooperative dual-hop relaying systems with beamforming over Nakagami-m fading channels. IEEE Trans. Wireless Commun. 8(8), 3950-3954 (2009)

5. HA Suraweera, GK Karagiannidis, Closed-form error analysis of the non-identical Nakagami-m relay fading channel. IEEE Commun. Lett. 12(4), 259-261 (2008)

6. YA Chau, KY Huang, Channel statistics and performance of cooperative selection diversity with dual-hop amplify-and-forward relay over Rayleigh fading channels. IEEE Trans. Wireless Commun. 7(5), 1779-1785 (2008)

7. SS Ikki, MH Ahmed, Performance of cooperative diversity using equal gain combining (EGC) over Nakagami-m fading channels. IEEE Trans. Wireless Commun. 8(2), 557-562 (2009)

8. S Alapattu, N Rajatheva, C Tellambura, Performance analysis of TDMA relay protocols over Nakagami-m fading. IEEE Trans. Vehicular Technol. 59(1), 93-104 (2010)

9. I Krikidis, JS Thompson, S Mclaughlin, N Goertz, Max-min relay selection for legacy amplify-and-forward systems with interference. IEEE Trans. Wireless Commun. 8(6), 3016-3027 (2009)

10. C Zhong, S Jin, K-K Wong, Dual-hop systems with noisy relay and interference-limited destination. IEEE Trans. Commun. 58(3), 764-768 (2010)

11. W Xu, J Zhang, P Zhang, Outage probability of two-hop fixed-gain relay with interference at the relay and destination. IEEE Commun. Lett. 15(6), 608-610 (2011)

12. SS Ikki, S Aïssa, Impact of imperfect channel estimation and co-channel interference on dual-hop relaying systems. IEEE Commun. Lett. 16(3), 324-327 (2012)

13. SS Ikki, S Aïssa, Impact of imperfect channel estimation and co-channel interference on regenerative cooperative networks. IEEE Wireless Commun. Lett. 1(5), 436-439 (2012)

14. HA Suraweera, DS Michalopoulos, C Yuen, Performance analysis of fixed gain relay systems with a single interference in Nakagami- $m$ fading channels. IEEE Trans. Vehicular Technol. 61(3), 1457-1463 (2012)

15. FS Al-Qahtani, TQ Duong, C Zhong, KA Qaraqe, H Alnuweiri, Performance analysis of dual-hop AF systems with interference in Nakagami-m fading channels. IEEE Signal Process. Lett. 18(8), 454-457 (2011)

16. H Yu, I-H Lee, GL stüber, Outage probability of decode-and-forward cooperative relaying systems with co-channel interference. IEEE Trans. Commun. 11(1), 266-274 (2012)

17. T Soithong, VA Aalo, GP Efthymoglou, C Chayawan, Outage analysis of multihop relay systems in interference-limited Nakagami-m fading channels. IEEE Trans. Vehicular Technol. 61(3), 1451-1457 (2012)

18. FS Al-Qahtani, J Yang, RM Radaydeh, H Alnuweiri, On the capacity of two-hop AF relaying in the presence of interference under Nakagami-m fading. IEEE Commun. Lett. 17(1), 19-22 (2013)

19. PL Yeoh, M Elkashlan, IB Collings, Exact and asymptotic SER of distributed TAS/MRC in MIMO relay networks. IEEE Trans. Wireless Commun. 10(3), 751-756 (2011)

20. PL Yeoh, M Elkashlan, IB Collings, MIMO relaying: distributed TAS/MRC in Nakagami-m fading. IEEE Trans. Commun. 59(10), 2678-2682 (2011)

21. M Elkashlan, PL Yeoh, N Yang, TQ Duong, C Leung, A comparison of two MIMO relaying protocols in Nakagami-m fading. IEEE Trans. Vehicular Technol. 61(3), 1416-1422 (2012)

22. N Yang, M Elkashlan, PL Yeoh, J Yuan, Multiuser MIMO relay networks in Nakagami-m fading channels. IEEE Trans. Commun. 60(11), 3298-3310 (2012)

23. C Zhong, H Suraweera, C Yuen, A Huang, Z Zhang, Outage probability of dual-hop multiple antenna AF relaying systems with interference. IEEE Trans. Commun. 61(1), 108-119(2013)
24. H Ding, C He, L Jiang, Performance analysis of fixed gain MIMO relay systems in the presence of co-channel interference. IEEE Commun. Lett. 16(7), 1133-1136 (2012)

25. H Phan, TQ Duong, M Elkashlan, H-J Zepernick, Beamforming amplify-and-forward relay networks with feedback delay and interference. IEEE Signal Process. Lett. 19(1), 16-19 (2012)

26. SS Ikki, S Aïssa, Performance analysis of two-way amplify-and-forward relaying in the presence of co-channel interferences. IEEE Trans. Commun. 60(4), 933-939 (2012)

27. SS Ikki, S Aïssa, Performance evaluation and optimization of dual-hop communication over Nakagami- $m$ fading channels in the presence of co-channel interferences. IEEE Commun. Lett. 16(8), 1149-1152 (2012)

28. DS Michalopoulos, HA Suraweera, GK Karagiannidis, R Schober, Amplifyand-forward relay selection with outdated channel state information, in Proceedings of IEEE GLOBECOM (Miami, FL, USA, 6-10 Dec 2010)

29. JL Vicario, A Bel, JA Lopez-Salcedo, G Seco, Opportunistic relay selection with outdated CSI: outage probability and diversity analysis. IEEE Trans. Wireless Commun. 8(6), 2872-2876 (2009)

30. Y Ma, D Zhang, A Leith, Z Wang, Error performance of transmit beamforming with delayed and limited feedback. IEEE Trans. Wireless Commun. 8(3), 1164-1170 (2009)

31. IS Gradshteyn, IM Ryzhik, Table of Integrals, Series, and Products, 7th edn. (Academic, New York, 2007)

32. MK Simon, M-S Alouini, Digital Communications over Fading Channels, 2nd edn. (Wiley, Hoboken, 2005)

33. RM Radaydeh, Impact of delayed arbitrary transmit antenna selection on the performance of rectangular QAM with receive $M R C$ in fading channels. IEEE Commun. Lett. 13(6), 390-392 (2009)

34. Y Chen, C Tellambura, Distribution function of selection combiner output in equally correlated Rayleigh, Rician, and Nakagami-m fading channels. IEEE Trans. Commun. 52(11), 1948-1956 (2004)

35. AM Mathai, RK Saxena, The H-function with Application in Statistics and Other Disciplines. (Wiley, New York, 1987)

36. IS Ansari, S Al-Ahmadi, F Yilmaz, M-S Alouini, H Yanikomeroglu, A new formula for the BER of binary modulations with dual-branch selection over generalized-K composite fading channels. IEEE Trans. Commun. 59(10), 2654-2658 (2011)

37. RM Radaydeh, M-S Alouini, On the performance of arbitrary transmit selection for threshold-based receive MRC with and without co-channe interference. IEEE Trans. Commun. 59(11), 3177-3191 (2011)

38. Z Chen, Asymptotic performance of transmit antenna selection with maximal-ratio combining for generalized selection criterion. IEEE Commun. Lett. 8(4), 247-249 (2004)

39. AP Prudnikov, YA Brychkov, Ol Marichev, Integrals and Series, 3rd vol. (Gordon and Breach Science, New York, 1990)

40. L Fan, X Lei, W Li, Exact closed-form expression for ergodic capacity of amplify-and-forward relaying in channel-noise-assisted cooperative networks with relay selection. IEEE Commun. Lett. 15(3), 332-333 (2011)

41. C Zhong, M Matthaiou, GK Karagiannidis, A Huang, Z Zhang, Capacity bounds for AF dual-hop relaying in $\mathrm{G}$ fading channels. IEEE Trans. Vehicular Technol. 61(4), 1730-1740 (2012)

doi:10.1186/1687-1499-2013-284

Cite this article as: Wang et al:: Performance analysis of interference-limited dual-hop multiple antenna AF relaying systems with feedback delay. EURASIP Journal on Wireless Communications and Networking 2013 2013:284 